\title{
On phases and interference of local communications in molecules
}

\author{
Roman F. Nalewajski
}

Received: 18 July 2013 / Accepted: 10 August 2013 / Published online: 13 September 2013

(C) The Author(s) 2013. This article is published with open access at Springerlink.com

\begin{abstract}
The role of phases in local Communication Theory of the Chemical Bond is investigated. Probability amplitudes of such molecular (fine-grained) information systems originate from the superposition principle of quantum mechanics involving the projection onto the bond system defined by the subspace of the state occupied Molecular Orbitals. They are explicitly phase-dependent, thus being capable of interference effects. The phase factors of the local direct and indirect (bridge, cascade) channels are examined and the associated amplitude/probability sum rules are established. The entropic descriptors of the local channels, providing the system "covalent" (communication-noise) and "ionic" (information-flow) components, are investigated using prototype one-electron systems. The competition between these informationtheoretic measures of the chemical bond covalency (electron delocalization) and ionicity (electron localization) is illustrated in $\mathrm{H}_{2}^{+}$and $\mathrm{H}_{2}$.
\end{abstract}

Keywords Amplitude phases - Chemical bonds - Covalent/ionic components . Entropic multiplicities · Information theory · Interference of communications · Local propagations $\cdot$ Molecular communications

\section{Introduction}

The information theory (IT) [1-8] has been successfully applied to explore the electron probabilities and patterns of chemical bonds in molecules, e.g., [9-20]. Both the electron probability distribution, determined by the wave-function modulus, and the system current density, related to the gradient of the wave-function phase, ultimately

\footnotetext{
R. F. Nalewajski $(\varangle)$

Department of Theoretical Chemistry, Jagiellonian University,

R. Ingardena 3, 30-060 Cracow, Poland

e-mail: nalewajs@chemia.uj.edu.pl
} 
contribute to the resultant (quantum) information content of molecular states. The former reveals the classical information content, while the latter determines its nonclassical (quantum) complement in the overall information measure [9,10,20-22].

It has been argued elsewhere that many classical problems of theoretical chemistry can be approached afresh using this novel IT perspective, e.g., [9-20]. The displacements of the classical information distribution in a molecule, relative to the promolecular reference consisting of its constituent non-bonded atoms, have been investigated [11-15,17-20,23-25] and the least biased partition of the molecular electron distribution into subsystem contributions, e.g., densities of bonded atoms, has been examined [11-13,26-33]. This IT approach has been shown to lead to the "stockholder" atomsin-molecules (AIM) of Hirshfeld [34].

The spatial localization of specific chemical bonds and electron localization in atoms and molecules has also been tackled. The non-additive Fisher information in the atomic orbital (AO) resolution has been used as the contra-gradience $(\mathrm{CG})$ criterion for localizing the bonding regions in molecules [11-20,35-37], while the related information density in the molecular orbital (MO) resolution has been shown $[11,38]$ to determine the vital ingredient of the electron-localization function (ELF) [39-41].

The communication theory of the chemical bond (CTCB), which uses the entropic descriptors of the molecular information (communication) channels in the AIM, orbital or local resolutions of the electron probability distribution, has also been developed [11-13,42-59]. The same bond descriptors have been used to provide the information-scattering perspective on the intermediate stages in the electron redistribution processes [60], including the atom "promotion" via the orbital hybridization [61], and the communication theory for the excited electron configurations has been developed [62]. The phenomenological description of equilibria in molecular subsystems has been also proposed [11,63-65], which formally resembles that developed in ordinary thermodynamics [66].

The IT approach introduces into the theory of electronic structure of molecular systems the novel entropy-representation [10-22], which complements the familiar energy-representation of the molecular quantum mechanics. Such a dual perspective parallels that known from the ordinary thermodynamics. It establishes the equivalent energy and entropy/information principles governing the molecular equilibria, provides a new unifying perspective on the molecular electronic structure, extends the variety of tools for probing chemical processes, and enriches the range of available descriptors of the bonding patterns in molecules. It also increases our understanding of the classical (intuitive) chemical concepts, e.g., the identity of AIM, bond localization, sources and measures of bond-order, its covalent/ionic composition, etc.

Entropic probes of molecular electronic structure have provided attractive tools for describing the chemical bond phenomenon in information terms. For an exploration of the chemical bond multiplicities in the orbital communication theory (OCT) [13,5759,67-70] it is vital to examine how the input information is propagated between $\mathrm{AO}$, the typical basis functions used to describe the bonding (occupied) MO subspace of SCF LCAO MO calculations. The molecular system can indeed be regarded as an information system determined by the communication network of the electronic conditional probabilities, in which the elementary "units", relevant to the resolution level in question, emit and/or receive the electron-allocation signals [13]. 
This classical information scattering and flow processes can be characterized by standard tools of the Shannon's theory of communication [3,4,7,8,11-13], which introduce a new class of descriptors of molecular "connectivities" between AIM. The molecular communication "noise", measured by the network average conditional entropy (scattered information), reflects the AO indeterminism in a molecule, and hence also the electron delocalization effect synonymous with the chemical covalency concept. The complementary bond component, chemical iconicity, is probed by the channel average mutual information (information flow) descriptor, which reflects the AO deterministic (localization) aspect of the probability propagation in a molecule. These two IT components complement each other: the more ionic (deterministic) is the molecular communication system, the less covalent (indeterministic) is its probability propagation in the given AO basis. This reflects the familiar competition between these two bond components.

The new (through-bridge) mechanism of the intermediate orbital communications (entropic interactions) in molecules has also been identified [71-76], which complements the direct, through-space bond contributions. The IT approach also covers changes in the bond pattern effected by chemical reactions [77,78]. The equivalence of the vertical (density-constrained) energy and entropy/information rules in quantum mechanics $[21,22,79,80]$ parallels that of the complementary energy and entropy principles of thermodynamics [66].

These communication descriptors have been derived from the classical information channels determined by the conditional probabilities of the AO events in the stationary (non-degenerate) molecular state, for which the spatial-phase component, and hence also the associated probability current, both identically vanish. The truly quantum channel, capable of the communication interference [76,79-81], calls for the information system of the probability-amplitude propagation, with the scattering amplitudes then explicitly depending on phases of the (complex) emitting and monitoring event-states. Examining the role of MO phases in local probability propagations in molecules is the main purpose of the present work.

Throughout the article the following tensor notation is used: $A$ denotes a scalar quantity, $\boldsymbol{A}$ stands for the row- or column-vector, and $\mathbf{A}$ represents a square or rectangular matrix. The logarithm of the Shannon information measure is taken to an arbitrary but fixed base. In keeping with the custom in works on IT the logarithm taken to base 2 corresponds to the information measured in bits (binary digits), while selecting $\log =\ln$ expresses the amount of information in nats (natural units): 1 nat = 1.44 bits.

\section{Orbital and local communications in molecules}

An exploration of the chemical bond system in molecular electronic states calls for the $\mathrm{AO}$ resolution determined by the basis functions $\chi=\left(\chi_{1}, \chi_{2}, \ldots, \chi_{m}\right)$ of typical SCF LCAO MO calculations. Within the familiar Hartree-Fock (HF) theory the molecular ground-state of $N$ electrons is defined by the occupied (bonding) subspace $\boldsymbol{\varphi}=\chi \mathbf{C}\left\{\varphi_{s}=\phi_{s} \xi_{s}, s=1,2, \ldots, N\right\}$ of the singly-occupied spin-MO (SMO), representing $N$-lowest one-particle states, which give rise to the associated Slater determinant: 


$$
\Psi(N)=\operatorname{det}[\varphi] \equiv\left|\varphi_{1}, \varphi_{2}, \ldots, \varphi_{N}\right|
$$

Here, $\varphi_{s}(\boldsymbol{r}, \sigma)=\phi_{s}(\boldsymbol{r}) \xi_{s}(\sigma), \phi_{s}$ denotes the spatial MO, and $\xi_{s}$ stands for one of the two admissible spin states of an electron: $\xi_{s} \in\{\alpha$ (spin-up), $\beta$ (spin-down) $\}$. In the analogous Kohn-Sham (KS) description such determinant of $N$-lowest KS orbitals, which usually provides quite an adequate description of the system chemical bonds (see e.g., [11-13]), defines the hypothetical state of non-interacting electrons, which generates the same electron density as does the (correlated) ground state of the real (interacting) system.

In OCT the orbital channels [3,4,7,8,11-13] propagate probabilities of the electron assignments to basis functions $\chi$ of the SCF LCAO MO calculations, via the network of the occupied SMO $\varphi$. The underlying conditional probabilities of the "output" orbital events $\chi$ ', given the "input" orbital events $\chi$,

$$
\begin{aligned}
\mathbf{P}\left(\chi^{\prime} \mid \chi\right) & =\left\{P\left(\chi_{j} \mid \chi_{i}\right) \equiv P(j \mid i) \equiv P_{i \rightarrow j}=|A(j \mid i)|^{2}=\left|A_{i \rightarrow j}\right|^{2}\right\}, \\
P(j \mid i) & =P(i, j) / p_{i} \equiv P_{i, j} / p_{i},
\end{aligned}
$$

result from the (bond-projected) superposition principle of quantum mechanics [82]. Here $P(i, j) \equiv P_{i, j}$ denotes the probability of the joint-event of simultaneously observing $\left|\chi_{i}\right\rangle$ and $\left|\chi_{j}\right\rangle$ in the molecular bond system, while $p_{i}=\sum_{j} P(i, j)=n_{i} / N$ represents the associated probability of $\left|\chi_{i}\right\rangle$ alone, with $n_{i}$ standing for the average $\mathrm{AO}$ occupation number. As also indicated above, these communications are generated by the magnitude (modulus) of the associated quantum (complex) amplitudes $\mathbf{A}\left(\chi^{\prime} \mid \chi\right)=\left\{A(j \mid i)=A_{i \rightarrow j}\right\}$, of the emitting (input) states $\boldsymbol{a}=|\chi\rangle=\left\{\left|\chi_{i}\right\rangle\right\}$ among the monitoring/receiving (output) states $\boldsymbol{b}=\left|\chi^{\prime}\right\rangle=\left\{\left|\chi_{j}\right\rangle\right\}$.

A deeper understanding of the molecular electronic structure ultimately calls for the continuous, local ("fine-grained") description [81,83], to complement the discrete, orbital ("coarse-grained") resolution adopted in OCT. The original orbitalcommunication development can be indeed naturally generalized into this extreme level of resolving electron distributions in molecules, when one examines the information propagations between infinitesimal volume elements around $r \in \Re$ in the channel input and $\boldsymbol{r}^{\prime} \in \mathfrak{R}^{\prime}$ in its output, respectively, with $\mathfrak{R}$ or $\mathfrak{R}^{\prime}$ containing the whole physical space of all admissible electron localizations.

This local description $[81,83]$, which we shall further develop in the present analysis, uses the basis functions $\{|\boldsymbol{r}\rangle\}$ of the familiar position representation of quantum mechanics, identified by the continuous labels of the spatial coordinates of an electron. They determine both the input $\boldsymbol{a}=\{|\boldsymbol{r}\rangle\}$ and output $\boldsymbol{b}=\left\{\left|\boldsymbol{r}^{\prime}\right\rangle\right\}$ of the local molecular channel determined by the relevant conditional probabilities,

$$
\left\{P\left(\boldsymbol{r}^{\prime} \mid \boldsymbol{r}\right)=P_{\boldsymbol{r} \rightarrow \boldsymbol{r}^{\prime}}=\left|A\left(\boldsymbol{r}^{\prime} \mid \boldsymbol{r}\right)\right|^{2}=\left|A_{\boldsymbol{r} \rightarrow \boldsymbol{r}^{\prime}}\right|^{2}\right\}, P\left(\boldsymbol{r}^{\prime} \mid \boldsymbol{r}\right)=\pi\left(\boldsymbol{r}, \boldsymbol{r}^{\prime}\right) / p(\boldsymbol{r}),
$$

where $\pi\left(\boldsymbol{r}, \boldsymbol{r}^{\prime}\right)=P_{\boldsymbol{r}, \boldsymbol{r}^{\prime}}=\left|A_{\boldsymbol{r}, \boldsymbol{r}^{\prime}}\right|^{2}$ again denotes the joint probability of simultaneously observing the two locations in the bond system of a molecule, and the associated probability of a single location, 


$$
p(\boldsymbol{r})=\rho(\boldsymbol{r}) / N=\int \pi\left(\boldsymbol{r}, \boldsymbol{r}^{\prime}\right) d \boldsymbol{r}^{\prime}
$$

represents the shape-factor of the molecular electron density $\rho(\boldsymbol{r})$.

In the simplest (one-determinantal) orbital approximation one takes into account only a single orbital configuration, e.g., the ground-state $\Psi(N)$ [Eq. (1)], the occupied SMO of which give rise to all physical properties of the system under consideration. This configuration is uniquely identified by its singly-occupied (physical) SMO subspace $\boldsymbol{\varphi}=\left(\varphi_{1}, \varphi_{2}, \ldots, \varphi_{N}\right)$, or by the associated spatial MO functions, $\boldsymbol{\phi}=\left(\phi_{1}, \phi_{2}, \ldots, \phi_{N}\right)=\chi \mathbf{C}$. They define the corresponding (idempotent) SMO and $\mathrm{MO}$ projectors onto this bond subspace,

$$
\hat{\mathrm{P}}_{\varphi} \equiv \sum_{s=1}^{N}\left|\phi_{s}\right\rangle\left\langle\phi_{s}|=| \varphi\right\rangle\langle\varphi|,\left(\hat{\mathrm{P}}_{\varphi}\right)^{2}=\hat{\mathrm{P}}_{\varphi} \quad \text { or } \quad \hat{\mathrm{P}}_{\varphi} \equiv|\boldsymbol{\phi}\rangle\langle\phi|, \quad\left(\hat{\mathrm{P}}_{\varphi}\right)^{2}=\hat{\mathrm{P}}_{\varphi}
$$

which generate the system charge-and-bond-order (CBO) matrix, i.e., the (idempotent) one-electron density matrix in the $\mathrm{AO}$ representation,

$$
\begin{aligned}
\gamma & =\langle\chi \mid \varphi\rangle\langle\varphi \mid \chi\rangle \equiv\left\langle\chi\left|\hat{\mathrm{P}}_{\varphi}\right| \chi\right\rangle=\langle\chi \mid \boldsymbol{\phi}\rangle\langle\boldsymbol{\phi} \mid \chi\rangle=\mathbf{C} \mathbf{C}^{\dagger}=\left\{\gamma_{i, j}=\boldsymbol{c}_{i} \boldsymbol{c}_{j}^{\dagger}\right\} \\
\operatorname{tr} \boldsymbol{\gamma} & \equiv \sum_{i} \gamma_{i, i}=N \sum_{i} p_{i} \equiv \sum_{i} n_{i}=N
\end{aligned}
$$

here the rectangular matrix of the LCAO MO expansion coefficients, $\mathbf{C}=\langle\chi \mid \varphi\rangle=$ $\langle\boldsymbol{\chi} \mid \boldsymbol{\phi}\rangle=\left\{\boldsymbol{C}_{s}\right\}=\left\{\boldsymbol{c}_{i}\right\}$ groups the column-vectors $\boldsymbol{C}_{s}=\left\{\boldsymbol{C}_{i, s}, i=1,2, \ldots, m\right\}$ defining $\phi_{s}=\chi \boldsymbol{C}_{s}$, i.e., the $s$ th column of $\mathbf{C}$, and row-vectors $\boldsymbol{c}_{i}=\left\{C_{i, s}, s=1,2, \ldots, N\right\}$, multiplying $\chi_{i}$ in all occupied MO combinations, i.e., the $i$ th row of $\mathbf{C}$. The density matrix idempotency,

$$
\begin{aligned}
\gamma^{2} & =\left\langle\chi\left|\hat{\mathrm{P}}_{\varphi}\right| \chi\right\rangle\left\langle\chi\left|\hat{\mathrm{P}}_{\varphi}\right| \chi\right\rangle=\left\langle\chi\left|\hat{\mathrm{P}}_{\varphi} \hat{\mathrm{P}}_{\chi} \hat{\mathrm{P}}_{\varphi}\right| \chi\right\rangle \\
& =\mathbf{C}\left(\mathbf{C}^{\dagger} \mathbf{C}\right) \mathbf{C}^{\dagger}=\mathbf{C} \mathbf{C}^{\dagger}=\gamma,
\end{aligned}
$$

follows from the assumned $\mathrm{AO}$ and $\mathrm{MO}$ orthonormalities,

$$
\langle\chi \mid \chi\rangle=\mathbf{I}_{m} \quad \text { and } \quad\langle\varphi \mid \varphi\rangle=\langle\boldsymbol{\phi} \mid \boldsymbol{\phi}\rangle=\langle\varphi \mid \chi\rangle\langle\chi \mid \chi\rangle\langle\chi \mid \varphi\rangle=\mathbf{C}^{\dagger} \mathbf{I}_{m} \mathbf{C}=\mathbf{C}^{\dagger} \mathbf{C}=\mathbf{I}_{N},
$$

which further imply [see Eq. (7)]:

$$
\hat{\mathrm{P}}_{\varphi} \hat{\mathrm{P}}_{\chi} \hat{\mathrm{P}}_{\varphi}=|\varphi\rangle\left(\mathbf{C}^{\dagger} \mathbf{C}\right)\langle\varphi|=\hat{\mathrm{P}}_{\varphi}
$$

Indeed, for the occupied MO $\varphi$ expressed in the given basis $\chi$, the action of the AO projector onto the occupied-MO projector amounts to identity operation, $\hat{\mathrm{P}}_{\chi}=$ $|\chi\rangle\langle\chi|=1$, and hence

$$
\hat{\mathrm{P}}_{\varphi} \hat{\mathrm{P}}_{\chi} \hat{\mathrm{P}}_{\varphi}=\left(\hat{\mathrm{P}}_{\varphi}\right)^{2}=\hat{\mathrm{P}}_{\varphi}
$$


(a) $\gamma_{i, j}=\left\langle\chi_{i} \mid \varphi\right\rangle\left\langle\varphi \mid \chi_{j}\right\rangle=\sum_{s} C_{i, s} C_{j, s}{ }^{*} \equiv \boldsymbol{c}_{i} \boldsymbol{c}_{j}^{\dagger} \equiv \Gamma_{i, j}$ input $\mid$ bond system $\mid$ output

(b) $\gamma\left(\boldsymbol{r}, \boldsymbol{r}^{\prime}\right)=\langle\boldsymbol{r} \mid \boldsymbol{\varphi}\rangle\left\langle\boldsymbol{\varphi} \mid \boldsymbol{r}^{\prime}\right\rangle=\sum_{s} \varphi_{s}(\boldsymbol{r}) \varphi_{s}^{*}\left(\boldsymbol{r}^{\prime}\right)=\boldsymbol{\varphi}(\boldsymbol{r}) \boldsymbol{\varphi}^{\dagger}\left(\boldsymbol{r}^{\prime}\right) \equiv \Gamma_{\boldsymbol{r}, \boldsymbol{r}^{\prime}}$ input $\mid$ bond system $\mid$ output

Fig. 1 Elementary amplitudes $\Gamma_{i, j}=\gamma_{i, j}$ (Panel a) and $\Gamma_{\boldsymbol{r}, \boldsymbol{r}^{\prime}}=\gamma\left(\boldsymbol{r}, \boldsymbol{r}^{\prime}\right)($ Panel $\boldsymbol{b}$ ), of the joint $t w o$-AO density $\Omega(i, j)=\left|\Gamma_{i, j}\right|^{2}$ and the joint $t w o$-point density $\Omega\left(\boldsymbol{r}, \boldsymbol{r}^{\prime}\right)=\left|\Gamma_{\boldsymbol{r}, \boldsymbol{r}^{\prime}}\right|^{2}$, measuring the AO and local representations of the SMO (bond) projection operator $\hat{\mathrm{P}}_{\varphi}: \gamma_{i, j}=\left\langle\chi_{i}\left|\hat{\mathrm{P}}_{\varphi}\right| \chi_{j}\right\rangle$ and $\gamma\left(\boldsymbol{r}, \boldsymbol{r}^{\prime}\right)=\left\langle\boldsymbol{r}\left|\hat{\mathrm{P}}_{\varphi}\right| \boldsymbol{r}^{\prime}\right\rangle$. Here, the row-vectors $\left\{\boldsymbol{c}_{i}\right\}$ combine the LCAO MO coefficients multiplying $\chi_{i}$ in $N$ occupied MO, collected as the $i$-th row of $\mathbf{C}$

The representative element $\gamma_{i, j}$ of the CBO matrix thus offers a transparent interpretation of amplitudes of the scattering density, which is shown in Fig. 1a. In this elementary two-orbital amplitude the ground-state $\mathrm{SMO}$ projector $\hat{\mathrm{P}}_{\varphi}$ represents the molecular bond system, which defines a network of molecular connections ("circuitry", "wiring" system), $\left|\chi_{i}\right\rangle$ specifies the input AO event of the signal origin, while the other orbital $\left|\chi_{j}\right\rangle$ states the output AO event of the signal monitoring/receiving.

In the one-electron Hilbert space the CBO matrix element has a simple "geometric" interpretation as the scalar product ("overlap") between the bond projected (non-orthogonal) $\mathrm{AO},\left|\chi^{b}\right\rangle=\hat{\mathrm{P}}_{\varphi}|\chi\rangle=\left\{\left|\chi_{i}^{b}\right\rangle=\hat{\mathrm{P}}_{\varphi}\left|\chi_{i}\right\rangle=\sum_{s}\left|\phi_{s}\right\rangle\left\langle\phi_{s} \mid \chi_{i}\right\rangle \equiv\right.$ $\left.\sum_{s}\left|\phi_{s}\right\rangle \phi_{s, i}^{*}=\sum_{s}\left|\phi_{s}\right\rangle C_{i, s}^{*}\right\}$ :

$$
\gamma_{i, j}=\left\langle\chi_{i}\left|\hat{\mathrm{P}}_{\varphi}\right| \chi_{i}\right\rangle=\left(\left\langle\chi_{i}\right| \hat{\mathrm{P}}_{\varphi}\right)\left(\hat{\mathrm{P}}_{\varphi}\left|\chi_{i}\right\rangle\right) \equiv\left\langle\chi_{i}^{b} \mid \chi_{j}^{b}\right\rangle \equiv S_{i, j}^{b}
$$

Indeed the bond-subspace $|\varphi\rangle$ has a lower dimensionality compared to that of the AO space, so that the non-orthogonal AO projections $\left|\chi^{b}\right\rangle$ effectively span only the $N$-dimensional (bond) subspace itself. Hence, a generally non-vanishing bond-overlap $S_{i, j}^{b}=\gamma_{i, j}$ in fact measures the (complex) projection of $\left|\chi_{j}^{b}\right\rangle$ onto $\left|\chi_{i}^{b}\right\rangle$.

The conditional probabilities of AO communications via its occupied SMO subspace [Eq. (2)] in this ground-state configuration,

$$
\mathbf{P}\left(\chi^{\prime} \mid \chi\right)=\left\{P(j \mid i)=\gamma_{i, j} \gamma_{j, i} / \gamma_{i, i}=\left|\Gamma_{i, j}\right|^{2} / n_{i} \equiv \Omega(i, j) / n_{i}\right\},
$$

also identify the associated joint-probabilities of AO pairs:

$$
\mathbf{P}\left(\chi^{\prime}, \chi\right)=\left\{P(i, j)=P_{i, j}=\Omega(i, j) / N=p_{i} P(j \mid i)=\gamma_{i, j} \gamma_{j, i} / N\right\}
$$

where the normalized AO probabilities $\boldsymbol{p}=\left\{p_{i}=\gamma_{i, i} / N \equiv n_{i} / N\right\}$ [see Eq. (6)]. It can be straightforwardly verified that these two-AO distributions also satisfy the expected normalizations: 


$$
\begin{aligned}
& \sum_{j} P(i, j)=(1 / N) \sum_{j} \gamma_{i, j} \gamma_{j, i}=n_{i} / N=p_{i}, \\
& \sum_{j} P(j \mid i)=\left(1 / n_{i}\right) \sum_{j} \gamma_{i, j} \gamma_{j, i}=n_{i} / n_{i}=1 .
\end{aligned}
$$

To summarize, the CBO matrix elements $\left\{\gamma_{i, j}\right\}$ directly measure the amplitudes $\left\{\Gamma_{i, j}\right\}$ of the AO-pair densities $\left\{\Omega(i, j)=\left|\Gamma_{i, j}\right|^{2}\right\}$ and the associated probabilities $\{P(i, j)=\Omega(i, j) / N\}$. The communication amplitudes are proportional to the corresponding (input-renormalized) CBO matrix elements:

$$
\mathbf{A}\left(\chi^{\prime} \mid \chi\right)=\left\{A(j \mid i)=A_{i \rightarrow j}=\gamma_{i, j} /\left(\gamma_{i, i}\right)^{1 / 2}=\Gamma_{i, j} /\left(n_{i}\right)^{1 / 2}\right\}
$$

The amplitudes $\left\{\Gamma_{i, j}\right\}$ and $\left\{A_{i \rightarrow j}\right\}$ of the signal propagation in molecular bond-system reflect both moduli and phases of $\left\{\gamma_{i, j}\right\}$, thus being capable of the communication "interference" [76,79,80].

The fine-grained approach adopts the local basis set of the precise localization states $\{|\boldsymbol{r}\rangle\}$ of an electron, in which the occupied SMO projector gives rise to the ordinary (idempotent) one-electron density matrix. For example, for the single, ground-state Slater determinant of Eq. (1)] in HF or KS theories one finds:

$$
\begin{aligned}
\gamma\left(\boldsymbol{r}, \boldsymbol{r}^{\prime}\right)=\langle\boldsymbol{r} \mid \boldsymbol{\varphi}\rangle\left\langle\boldsymbol{\varphi} \mid \boldsymbol{r}^{\prime}\right\rangle & =\left\langle\boldsymbol{r}\left|\hat{\mathrm{P}}_{\varphi}\right| \boldsymbol{r}^{\prime}\right\rangle=\sum_{s} \phi_{S}(\boldsymbol{r}) \phi_{S}\left(\boldsymbol{r}^{\prime}\right)^{*} \equiv \Gamma_{\boldsymbol{r}, \boldsymbol{r}^{\prime}} \\
& =\left(\langle\boldsymbol{r}| \hat{\mathrm{P}}_{\boldsymbol{\varphi}}\right)\left(\hat{\mathrm{P}}_{\boldsymbol{\varphi}}\left|\boldsymbol{r}^{\prime}\right\rangle\right) \equiv\left(\left\langle\boldsymbol{r}^{b} \mid \boldsymbol{r}^{\prime}\right\rangle \equiv S_{\boldsymbol{r}, \boldsymbol{r}^{\prime}}^{b},\right. \\
\operatorname{tr} \gamma\left(\boldsymbol{r}, \boldsymbol{r}^{\prime}\right) & \equiv \int \gamma(\boldsymbol{r}, \boldsymbol{r}) d \boldsymbol{r}=\int \rho(\boldsymbol{r}) d \boldsymbol{r}=N, \\
\int \gamma\left(\boldsymbol{r}, \boldsymbol{r}^{\prime}\right) \gamma\left(\boldsymbol{r}^{\prime}, \boldsymbol{r}^{\prime \prime}\right) d \boldsymbol{r}^{\prime} & =\langle\boldsymbol{r} \mid \boldsymbol{\phi}\rangle \int\left\langle\boldsymbol{\phi} \mid \boldsymbol{r}^{\prime}\right\rangle\left\langle\boldsymbol{r}^{\prime} \mid \boldsymbol{\phi}\right\rangle d \boldsymbol{r}^{\prime}\left\langle\boldsymbol{\phi} \mid \boldsymbol{r}^{\prime \prime}\right\rangle \\
& =\langle\boldsymbol{r} \mid \boldsymbol{\phi}\rangle\langle\boldsymbol{\phi} \mid \boldsymbol{\phi}\rangle\left\langle\boldsymbol{\phi} \mid \boldsymbol{r}^{\prime \prime}\right\rangle=\langle\boldsymbol{r} \mid \boldsymbol{\phi}\rangle\left\langle\boldsymbol{\phi} \mid \boldsymbol{r}^{\prime \prime}\right\rangle=\gamma\left(\boldsymbol{r}, \boldsymbol{r}^{\prime \prime}\right) .
\end{aligned}
$$

Above, we have used the MO orthonormality of Eq. (8), $\rho(\boldsymbol{r})=N p(\boldsymbol{r})$ stands for the electron density, while $p(\boldsymbol{r})=\gamma(\boldsymbol{r}, \boldsymbol{r}) / N$ denotes its "shape" (probability) factor [see Eq. (4)]. The elementary amplitude $\Gamma_{\boldsymbol{r}, \boldsymbol{r}^{\prime}}$ of the joint $t w o$-point density $\Omega\left(\boldsymbol{r}, \boldsymbol{r}^{\prime}\right)=\left|\Gamma_{\boldsymbol{r}, \boldsymbol{r}^{\prime}}\right|^{2}$ is thus directly determined by $\gamma\left(\boldsymbol{r}, \boldsymbol{r}^{\prime}\right)$ (see Fig. 1b), while the associated two-point probability distribution $\pi\left(\boldsymbol{r}, \boldsymbol{r}^{\prime}\right)=\Omega\left(\boldsymbol{r}, \boldsymbol{r}^{\prime}\right) / N$. The relevant normalization conditions are summarized in two preceding equations. We have also given in Eq. (16) the geometric interpretation of the $\gamma\left(\boldsymbol{r}, \boldsymbol{r}^{\prime}\right)$ kernel in molecular Hilbert space, as the bond-overlap $S_{\boldsymbol{r}, \boldsymbol{r}^{\prime}}^{b}=\left\langle\boldsymbol{r}^{b} \mid \boldsymbol{r}^{\prime}\right\rangle$ measuring the scalar product between the (non-orthogonal) bond-projected local basis vectors $\left\{\left|\boldsymbol{r}^{b}\right\rangle=\hat{\mathrm{P}}_{\varphi}|\boldsymbol{r}\rangle\right\}$.

The local information system involves these strict localization events in both its input $\boldsymbol{a}=\{|\boldsymbol{r}\rangle\}$ and output $\boldsymbol{b}=\left\{\left|\boldsymbol{r}^{\prime}\right\rangle\right\}$. The elementary communication amplitude is then determined by the locally renormalized kernel of Eq. (16) and Fig. 1b,

$$
A\left(\boldsymbol{r}^{\prime} \mid \boldsymbol{r}\right)=A_{\boldsymbol{r} \rightarrow \boldsymbol{r}^{\prime}}=\gamma\left(\boldsymbol{r}, \boldsymbol{r}^{\prime}\right) /[\gamma(\boldsymbol{r}, \boldsymbol{r})]^{1 / 2}=\Gamma_{\boldsymbol{r}, \boldsymbol{r}^{\prime}} /[\rho(\boldsymbol{r})]^{1 / 2},
$$


which ultimately determines the associated conditional probability between the two electron-localization states,

$$
\begin{aligned}
P\left(\boldsymbol{r}^{\prime} \mid \boldsymbol{r}\right) & =P_{\boldsymbol{r} \rightarrow \boldsymbol{r}^{\prime}}=\left|\Gamma_{\boldsymbol{r}, \boldsymbol{r}^{\prime}}\right|^{2} / \rho(\boldsymbol{r})=\left|A_{\boldsymbol{r} \rightarrow \boldsymbol{r}^{\prime}}\right|^{2}=\gamma\left(\boldsymbol{r}, \boldsymbol{r}^{\prime}\right) \gamma\left(\boldsymbol{r}^{\prime}, \boldsymbol{r}\right) / \gamma(\boldsymbol{r}, \boldsymbol{r}) \\
& =\Omega\left(\boldsymbol{r}, \boldsymbol{r}^{\prime}\right) / \rho(\boldsymbol{r}),
\end{aligned}
$$

and the joint $t w o$-point probability distribution:

$$
\pi\left(\boldsymbol{r}, \boldsymbol{r}^{\prime}\right)=p(\boldsymbol{r}) P\left(\boldsymbol{r}^{\prime} \mid \boldsymbol{r}\right)=\Omega\left(\boldsymbol{r}, \boldsymbol{r}^{\prime}\right) / N
$$

One directly verifies the required normalizations of these two-point probability distributions using the idempotency property of the density matrix [Eq. (17)]:

$$
\begin{aligned}
& \int \pi\left(\boldsymbol{r}^{\prime}, \boldsymbol{r}^{\prime}\right) d \boldsymbol{r}^{\prime}=(1 / N) \int \Omega\left(\boldsymbol{r}, \boldsymbol{r}^{\prime}\right) d \boldsymbol{r}^{\prime}=\gamma(\boldsymbol{r}, \boldsymbol{r}) / N=p(\boldsymbol{r}), \\
& \int P\left(\boldsymbol{r}^{\prime} \mid \boldsymbol{r}\right) d \boldsymbol{r}^{\prime}=[\rho(\boldsymbol{r})]^{-1} \int \Omega\left(\boldsymbol{r}, \boldsymbol{r}^{\prime}\right) d \boldsymbol{r}^{\prime}=\gamma(\boldsymbol{r}, \boldsymbol{r}) / \rho(\boldsymbol{r})=1 .
\end{aligned}
$$

Therefore, the density-matrix kernel $\gamma\left(\boldsymbol{r}, \boldsymbol{r}^{\prime}\right)$ fully determines all local communications between the system infinitesimal volume-elements via the subspace of the configuration occupied SMO:

$$
\left\{\pi\left(\boldsymbol{r}, \boldsymbol{r}^{\prime}\right)=P_{\boldsymbol{r}, \boldsymbol{r}^{\prime}}[\gamma]\right\},\left\{P\left(\boldsymbol{r}^{\prime} \mid \boldsymbol{r}\right)=P_{\boldsymbol{r} \rightarrow \boldsymbol{r}^{\prime}}[\gamma]\right\} \quad \text { and } \quad\left\{A\left(\boldsymbol{r}^{\prime} \mid \boldsymbol{r}\right)=A_{\boldsymbol{r} \rightarrow \boldsymbol{r}^{\prime}}[\gamma]\right\}
$$

While the local conditional probability $P\left(\boldsymbol{r}^{\prime} \mid \boldsymbol{r}\right)$ is independent of the spatial phase of the "off-diagonal" part of $\gamma\left(\boldsymbol{r}, \boldsymbol{r}^{\prime}\right)$, for $\boldsymbol{r}^{\prime} \neq \boldsymbol{r}$, which determines the non-local communications $\left\{|\boldsymbol{r}\rangle \rightarrow\left|\boldsymbol{r}^{\prime}\right\rangle\right\}$, its amplitude $A\left(\boldsymbol{r}^{\prime} \mid \boldsymbol{r}\right)$ is seen to be explicitly dependent upon the relative phase of MO in the two locations involved, which also determines the resultant phase of $\gamma\left(\boldsymbol{r}, \boldsymbol{r}^{\prime}\right)$. Clearly, this dependence vanishes for the "diagonal" part of $\gamma\left(\boldsymbol{r}, \boldsymbol{r}^{\prime}\right)$, i.e., the local communications $\{|\boldsymbol{r}\rangle \rightarrow|\boldsymbol{r}\rangle\}$, when

$$
\left\{\pi(\boldsymbol{r}, \boldsymbol{r})=\rho(\boldsymbol{r})^{2} / N, \quad P(\boldsymbol{r} \mid \boldsymbol{r})=\rho(\boldsymbol{r}), \quad A(\boldsymbol{r} \mid \boldsymbol{r})=\rho(\boldsymbol{r})^{1 / 2}\right\} .
$$

One also observes, that both locations of an electron enter symmetrically into the joint probabilities $\pi\left(\boldsymbol{r}, \boldsymbol{r}^{\prime}\right)$ and the associated density distribution $\Omega\left(\boldsymbol{r}, \boldsymbol{r}^{\prime}\right)$, while in the directed conditional probabilities $P_{\boldsymbol{r} \rightarrow \boldsymbol{r}^{\prime}}$ and $P_{\boldsymbol{r}^{\prime} \rightarrow \boldsymbol{r}}$ or in the associated amplitudes $A_{\boldsymbol{r} \rightarrow \boldsymbol{r}^{\prime}}$ and $A_{\boldsymbol{r}^{\prime} \rightarrow \boldsymbol{r}}$ the two position labels are non-symmetrical with respect to their exchange ("transposition"), as explicitly indicated by the arrow specifying the direction of the underlying communication: $\boldsymbol{r} \rightarrow \boldsymbol{r}^{\prime}$ or $\boldsymbol{r}^{\prime} \rightarrow \boldsymbol{r}$. However, one could also define the average communications between the two locations, $\boldsymbol{r} \leftrightarrow \boldsymbol{r}^{\prime}$, without any specific reference to their directions, by combining the "forward" $\left(\boldsymbol{r} \rightarrow \boldsymbol{r}^{\prime}\right)$ and "reverse" ( $\left.\boldsymbol{r}^{\prime} \rightarrow \boldsymbol{r}\right)$ communications for the two specified locations of an electron. This defines the symmetric communication links and the associated amplitudes:

$$
\left\{P\left(\boldsymbol{r} \leftrightarrow \boldsymbol{r}^{\prime}\right)=P_{\boldsymbol{r} \leftrightarrow \boldsymbol{r}^{\prime}}=\left[P\left(\boldsymbol{r}^{\prime} \mid \boldsymbol{r}\right)+P\left(\boldsymbol{r} \mid \boldsymbol{r}^{\prime}\right)\right] / 2=\pi\left(\boldsymbol{r}, \boldsymbol{r}^{\prime}\right) /\left[2 \rho_{h}\left(\boldsymbol{r}, \boldsymbol{r}^{\prime}\right)\right] \equiv\left(A_{\boldsymbol{r} \leftrightarrow \boldsymbol{r}^{\prime}}\right)^{2} .\right.
$$


Above, the harmonic average $\rho_{h}\left(\boldsymbol{r}, \boldsymbol{r}^{\prime}\right)$ of densities in the two specified electron positions is defined by the relation:

$$
\rho_{h}\left(\boldsymbol{r}, \boldsymbol{r}^{\prime}\right)^{-1} \equiv \rho(\boldsymbol{r})^{-1}+\rho\left(\boldsymbol{r}^{\prime}\right)^{-1} \text { or } \rho_{h}\left(\boldsymbol{r}, \boldsymbol{r}^{\prime}\right)=\rho(\boldsymbol{r}) \rho\left(\boldsymbol{r}^{\prime}\right) /\left[\rho(\boldsymbol{r})+\rho\left(\boldsymbol{r}^{\prime}\right)\right]
$$

It reflects the ratio between the square of the associated geometric average, $\rho_{g}\left(\boldsymbol{r}, \boldsymbol{r}^{\prime}\right) \equiv$ $\left[\rho(\boldsymbol{r}) \rho\left(\boldsymbol{r}^{\prime}\right)\right]^{1 / 2}$, and the arithmetic average $\rho_{a}\left(\boldsymbol{r}, \boldsymbol{r}^{\prime}\right) \equiv\left[\rho(\boldsymbol{r})+\rho\left(\boldsymbol{r}^{\prime}\right)\right] / 2=N p_{a}\left(\boldsymbol{r}, \boldsymbol{r}^{\prime}\right)$ of the two densities:

$$
2 \rho_{h}\left(\boldsymbol{r}, \boldsymbol{r}^{\prime}\right)=2 \rho(\boldsymbol{r}) \rho\left(\boldsymbol{r}^{\prime}\right) /\left[\rho(\boldsymbol{r})+\rho\left(\boldsymbol{r}^{\prime}\right)\right]=\rho_{g}\left(\boldsymbol{r}, \boldsymbol{r}^{\prime}\right)^{2} / \rho_{a}\left(\boldsymbol{r}, \boldsymbol{r}^{\prime}\right) \geq 0
$$

Hence, the symmetric (non-directional, positive) amplitude of such mutual communications between the two specified locations reads:

$$
\begin{aligned}
A_{\boldsymbol{r} \leftrightarrow \boldsymbol{r}^{\prime}} & =\left[\gamma_{g}\left(\boldsymbol{r}, \boldsymbol{r}^{\prime}\right) / \rho_{g}\left(\boldsymbol{r}, \boldsymbol{r}^{\prime}\right)\right] p_{a}\left(\boldsymbol{r}, \boldsymbol{r}^{\prime}\right)^{1 / 2}, \\
\gamma_{g}\left(\boldsymbol{r}, \boldsymbol{r}^{\prime}\right) & =\Omega\left(\boldsymbol{r}, \boldsymbol{r}^{\prime}\right)^{1 / 2}=\left|\gamma\left(\boldsymbol{r}, \boldsymbol{r}^{\prime}\right)\right|, \\
p_{a}\left(\boldsymbol{r}, \boldsymbol{r}^{\prime}\right) & =\rho_{a}\left(\boldsymbol{r}, \boldsymbol{r}^{\prime}\right) / N .
\end{aligned}
$$

\section{Phases in local direct and cascade communications}

Consider first the direct communication $\boldsymbol{r} \rightarrow \boldsymbol{r}^{\prime}$ between the two locations in a molecule, defined by the amplitude of Eq. (18), the phase of which is determined by that of the amplitude $\Gamma_{\boldsymbol{r}, \boldsymbol{r}^{\prime}}=\gamma\left(\boldsymbol{r}, \boldsymbol{r}^{\prime}\right)$ of the joint $t w o$-point distribution [Eq. (16)]. Assuming generally complex $\mathrm{MO}$,

$$
\phi_{S}(\boldsymbol{r})=R_{s}(\boldsymbol{r}) \exp \left[\mathrm{i} \Phi_{S}(\boldsymbol{r})\right], \quad s=1,2, \ldots, N,
$$

one obtains the following expression for the density-matrix kernel:

$$
\begin{aligned}
\gamma\left(\boldsymbol{r}, \boldsymbol{r}^{\prime}\right) & =\sum_{s} \phi_{S}(\boldsymbol{r}) \phi_{S}\left(\boldsymbol{r}^{\prime}\right)^{*}=\sum_{s} R_{S}(\boldsymbol{r}) R_{S}\left(\boldsymbol{r}^{\prime}\right) \exp \left\{\mathrm{i}\left[\Phi_{S}(\boldsymbol{r})-\Phi_{S}\left(\boldsymbol{r}^{\prime}\right)\right]\right\} \\
& \equiv \sum_{s} m_{S}\left(\boldsymbol{r}, \boldsymbol{r}^{\prime}\right) \exp \left\{\mathrm{i} f_{S}\left(\boldsymbol{r}, \boldsymbol{r}^{\prime}\right)\right\} \equiv M\left(\boldsymbol{r}, \boldsymbol{r}^{\prime}\right) \exp \left\{\mathrm{i} F\left(\boldsymbol{r}, \boldsymbol{r}^{\prime}\right)\right\}
\end{aligned}
$$

where the resultant modulus $M\left(\boldsymbol{r}, \boldsymbol{r}^{\prime}\right)$ and phase $F\left(\boldsymbol{r}, \boldsymbol{r}^{\prime}\right)$ parts of $\gamma\left(\boldsymbol{r}, \boldsymbol{r}^{\prime}\right)$, representing the bond system as a whole, depend upon the constituent MO distributions: $\left\{m_{s}\left(\boldsymbol{r}, \boldsymbol{r}^{\prime}\right)\right\}$ and $\left\{f_{s}\left(\boldsymbol{r}, \boldsymbol{r}^{\prime}\right)\right\}$. The latter represent relative MO phases in two locations, $\left\{f_{s}\left(\boldsymbol{r}, \boldsymbol{r}^{\prime}\right)=\right.$ $\left.\Phi_{S}(\boldsymbol{r})-\Phi_{S}\left(\boldsymbol{r}^{\prime}\right)\right\}$, which identically vanish for the diagonal scattering $\boldsymbol{r} \rightarrow \boldsymbol{r}$, when $\gamma(\boldsymbol{r}, \boldsymbol{r})=\sum_{S}\left[R_{S}(\boldsymbol{r})\right]^{2}=M(\boldsymbol{r}, \boldsymbol{r})=\rho(\boldsymbol{r})$.

Next we examine the indirect, multi-stage (bridge, cascade) communications in molecular bond systems. The simplest, single-cascade scattering (Fig. 2a), between the terminal points $\boldsymbol{r}$ (input) and $\boldsymbol{r}^{\prime}$ (output) involve a single intermediate location $\boldsymbol{r}^{\prime \prime}=\boldsymbol{r}^{(1)}$. The relevant joint distribution amplitude $\Gamma\left(\boldsymbol{r}, \boldsymbol{r}^{\prime} ; \boldsymbol{r}^{\prime \prime}\right)$ for such a three-point 

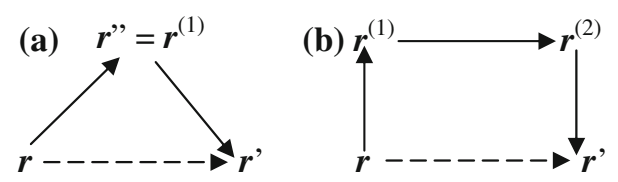

Fig. 2 Direct (broken arrows) and indirect (solid arrows) scattering between terminal locations $\boldsymbol{r}$ (input) and $\boldsymbol{r}^{\prime}$ (output), in the single- (Panel a) and double-(Panel b) cascades, involving the single $\left\{\boldsymbol{r}^{(1)}\right\}$ and double $\left\{\boldsymbol{r}^{(1)}, \boldsymbol{r}^{(2)}\right\}$ intermediate points (bridges), respectively

cascade propagation $\boldsymbol{r} \rightarrow \boldsymbol{r}^{(1)} \rightarrow \boldsymbol{r}^{\prime}$ is given by the product of the two amplitudes for each direct scattering stage:

$$
\begin{aligned}
\Gamma\left(\boldsymbol{r}, \boldsymbol{r}^{\prime} ; \boldsymbol{r}^{\prime \prime}\right) & =\Gamma_{\boldsymbol{r}, \boldsymbol{r}^{\prime \prime}} \Gamma_{\boldsymbol{r}^{\prime \prime}, \boldsymbol{r}^{\prime}}=\gamma\left(\boldsymbol{r}, \boldsymbol{r}^{\prime \prime}\right) \gamma\left(\boldsymbol{r}^{\prime \prime}, \boldsymbol{r}^{\prime}\right) \\
& =M\left(\boldsymbol{r}, \boldsymbol{r}^{\prime \prime}\right) M\left(\boldsymbol{r}^{\prime \prime}, \boldsymbol{r}^{\prime}\right) \exp \left\{\mathrm{i}\left[F\left(\boldsymbol{r}, \boldsymbol{r}^{\prime \prime}\right)+F\left(\boldsymbol{r}^{\prime \prime}, \boldsymbol{r}^{\prime}\right)\right]\right\} \\
& \equiv M\left(\boldsymbol{r}, \boldsymbol{r}^{\prime} ; \boldsymbol{r}^{\prime \prime}\right) \exp \left[\mathrm{i} F\left(\boldsymbol{r}, \boldsymbol{r}^{\prime} ; \boldsymbol{r}^{\prime \prime}\right)\right] .
\end{aligned}
$$

Integrating this indirect amplitude contribution over all admissible intermediate positions [see Eq. (17)] recovers the direct amplitude:

$$
\int \Gamma\left(\boldsymbol{r}, \boldsymbol{r}^{\prime} ; \boldsymbol{r}^{\prime \prime}\right) d \boldsymbol{r}^{\prime \prime}=\int \gamma\left(\boldsymbol{r}, \boldsymbol{r}^{\prime \prime}\right) \gamma\left(\boldsymbol{r}^{\prime \prime}, \boldsymbol{r}^{\prime}\right) d \boldsymbol{r}^{\prime \prime}=\gamma\left(\boldsymbol{r}, \boldsymbol{r}^{\prime}\right) .
$$

The resultant modulus in Eq. (30) is given by the product of moduli for each direct scattering in the bridge propagation,

$$
M\left(\boldsymbol{r}, \boldsymbol{r}^{\prime} ; \boldsymbol{r}^{\prime \prime}\right)=M\left(\boldsymbol{r}, \boldsymbol{r}^{\prime \prime}\right) M\left(\boldsymbol{r}^{\prime \prime}, \boldsymbol{r}^{\prime}\right),
$$

while the resultant phase is determined by the sum or relative phases of the two direct stages involved:

$$
F\left(\boldsymbol{r}, \boldsymbol{r}^{\prime} ; \boldsymbol{r}^{\prime \prime}\right)=F\left(\boldsymbol{r}, \boldsymbol{r}^{\prime \prime}\right)+F\left(\boldsymbol{r}^{\prime \prime}, \boldsymbol{r}^{\prime}\right)
$$

The product of two communication amplitudes at each stage of the through-bridge signal propagation then determines the resultant indirect amplitude,

$$
\begin{aligned}
A\left(\boldsymbol{r} \rightarrow \boldsymbol{r}^{\prime} ; \boldsymbol{r}^{\prime \prime}\right) & =A_{\boldsymbol{r} \rightarrow \boldsymbol{r}^{\prime \prime}} A_{\boldsymbol{r}^{\prime \prime} \rightarrow \boldsymbol{r}^{\prime}}=\Gamma_{\boldsymbol{r}, \boldsymbol{r}^{\prime \prime}} \Gamma_{\boldsymbol{r}^{\prime \prime}, \boldsymbol{r}^{\prime}} /\left[\rho(\boldsymbol{r}) \rho\left(\boldsymbol{r}^{\prime \prime}\right)\right]^{1 / 2}=\Gamma\left(\boldsymbol{r}, \boldsymbol{r}^{\prime} ; \boldsymbol{r}^{\prime \prime}\right) / \rho_{g}\left(\boldsymbol{r}, \boldsymbol{r}^{\prime \prime}\right) \\
& =\left[M\left(\boldsymbol{r}, \boldsymbol{r}^{\prime} ; \boldsymbol{r}^{\prime \prime}\right) / \rho_{g}\left(\boldsymbol{r}, \boldsymbol{r}^{\prime \prime}\right)\right] \exp \left[\mathrm{i} F\left(\boldsymbol{r}, \boldsymbol{r}^{\prime} ; \boldsymbol{r}^{\prime \prime}\right)\right],
\end{aligned}
$$

the square of which gives rise to the associated resultant conditional probability of the local single-cascade channel:

$$
P\left(\boldsymbol{r} \rightarrow \boldsymbol{r}^{\prime} ; \boldsymbol{r}^{\prime \prime}\right)=\left|A\left(\boldsymbol{r}, \boldsymbol{r}^{\prime} ; \boldsymbol{r}^{\prime \prime}\right)\right|^{2}=P_{\boldsymbol{r} \rightarrow \boldsymbol{r}^{\prime \prime}} P_{\boldsymbol{r}^{\prime \prime} \rightarrow \boldsymbol{r}^{\prime}}
$$

Its bridge-normalization reads [73]:

$$
\int d \boldsymbol{r}^{\prime \prime}\left[\int d \boldsymbol{r}^{\prime} P\left(\boldsymbol{r} \rightarrow \boldsymbol{r}^{\prime} ; \boldsymbol{r}^{\prime \prime}\right)\right]=\int d \boldsymbol{r}^{\prime \prime} P\left(\boldsymbol{r}^{\prime \prime} \mid \boldsymbol{r}\right)=1
$$


It should be observed that the phase factor of $A\left(\boldsymbol{r} \rightarrow \boldsymbol{r}^{\prime} ; \boldsymbol{r}^{\prime \prime}\right)$, determined by that of $\Gamma\left(\boldsymbol{r}, \boldsymbol{r}^{\prime} ; \boldsymbol{r}^{\prime \prime}\right)$ [Eq. (30)], disappears in $P\left(\boldsymbol{r} \rightarrow \boldsymbol{r}^{\prime} ; \boldsymbol{r}^{\prime \prime}\right)$. This is not the case in the parallel cascades generated by the multi-determinantal wave functions of the configurationinteraction $(\mathrm{CI})$ theory $[44,80,81]$.

These single-cascade results can be straightforwardly generalized into any bridgeorder. Consider, e.g., the double-cascade of Fig. 2b, in which the indirect signal propagation involves two intermediate points $\boldsymbol{r}^{(1)}$ and $\boldsymbol{r}^{(2)}$. Its joint distribution amplitude $\Omega\left(\boldsymbol{r}, \boldsymbol{r}^{\prime} ; \boldsymbol{r}^{(1)}, \boldsymbol{r}^{(2)}\right)$ for the double-cascade propagation $\boldsymbol{r} \rightarrow\left[\boldsymbol{r}^{(1)} \rightarrow \boldsymbol{r}^{(2)}\right] \rightarrow \boldsymbol{r}^{\prime}$ is now given by the product of three direct amplitudes:

$$
\begin{aligned}
\Gamma\left(\boldsymbol{r}, \boldsymbol{r}^{\prime} ; \boldsymbol{r}^{(1)}, \boldsymbol{r}^{(2)}\right)= & \gamma\left(\boldsymbol{r}, \boldsymbol{r}^{(1)}\right) \gamma\left(\boldsymbol{r}^{(1)}, \boldsymbol{r}^{(2)}\right) \gamma\left(\boldsymbol{r}^{(2)}, \boldsymbol{r}^{\prime}\right) \\
= & M\left(\boldsymbol{r}, \boldsymbol{r}^{(1)}\right) M\left(\boldsymbol{r}^{(1)}, \boldsymbol{r}^{(2)}\right) M\left(\boldsymbol{r}^{(2)}, \boldsymbol{r}^{\prime}\right) \exp \left\{\mathrm { i } \left[F\left(\boldsymbol{r}, \boldsymbol{r}^{(1)}\right)\right.\right. \\
& \left.\left.+F\left(\boldsymbol{r}^{(1)}, \boldsymbol{r}^{(2)}\right)+F\left(\boldsymbol{r}^{(2)}, \boldsymbol{r}^{\prime}\right)\right]\right\} \\
\equiv & M\left(\boldsymbol{r}, \boldsymbol{r}^{\prime} ; \boldsymbol{r}^{(1)}, \boldsymbol{r}^{(2)}\right) \exp \left[\mathrm{i} F\left(\boldsymbol{r}, \boldsymbol{r}^{\prime} ; \boldsymbol{r}^{(1)}, \boldsymbol{r}^{(2)}\right)\right] .
\end{aligned}
$$

Again, the integration over the two intermediate outputs $\boldsymbol{r}^{(1)}$ and $\boldsymbol{r}^{(2)}$ recovers the direct amplitude:

$$
\int d \boldsymbol{r}^{(1)} \int d \boldsymbol{r}^{(2)} \Gamma\left(\boldsymbol{r}, \boldsymbol{r}^{\prime} ; \boldsymbol{r}^{(1)}, \boldsymbol{r}^{(2)}\right)=\int d \boldsymbol{r}^{(1)} \gamma\left(\boldsymbol{r}, \boldsymbol{r}^{(1)}\right) \gamma\left(\boldsymbol{r}^{(1)}, \boldsymbol{r}^{\prime}\right)=\gamma\left(\boldsymbol{r}, \boldsymbol{r}^{\prime}\right)
$$

The associated communications are now determined by the double-bridge amplitude:

$$
\begin{aligned}
A\left(\boldsymbol{r} \rightarrow \boldsymbol{r}^{\prime} ; \boldsymbol{r}^{(1)}, \boldsymbol{r}^{(2)}\right) & =A\left(\boldsymbol{r}^{(1)} \mid \boldsymbol{r}\right) A\left(\boldsymbol{r}^{(2)} \mid \boldsymbol{r}^{(1)}\right) A\left(\boldsymbol{r}^{\prime} \mid \boldsymbol{r}^{(2)}\right)=\Gamma\left(\boldsymbol{r}, \boldsymbol{r}^{\prime} ; \boldsymbol{r}^{(1)}, \boldsymbol{r}^{(2)}\right) /\left[\rho(\boldsymbol{r}) \rho\left(\boldsymbol{r}^{(1)}\right) \rho\left(\boldsymbol{r}^{(2)}\right)\right]^{1 / 2}, \\
& =\left\{M\left(\boldsymbol{r}, \boldsymbol{r}^{\prime} ; \boldsymbol{r}^{(1)}, \boldsymbol{r}^{(2)}\right) /\left[\rho(\boldsymbol{r}) \rho\left(\boldsymbol{r}^{(1)}\right) \rho\left(\boldsymbol{r}^{(2)}\right)\right]^{1 / 2}\right\} \exp \left[\mathrm{i} F\left(\boldsymbol{r}, \boldsymbol{r}^{\prime} ; \boldsymbol{r}^{(1)}, \boldsymbol{r}^{(2)}\right)\right] .
\end{aligned}
$$

It generates the associated conditional probabilities,

$$
P\left(\boldsymbol{r} \rightarrow \boldsymbol{r}^{\prime} ; \boldsymbol{r}^{(1)}, \boldsymbol{r}^{(2)}\right)=\left|A\left(\boldsymbol{r} \rightarrow \boldsymbol{r}^{\prime} ; \boldsymbol{r}^{(1)}, \boldsymbol{r}^{(2)}\right)\right|^{2}=\left[M\left(\boldsymbol{r}, \boldsymbol{r}^{\prime} ; \boldsymbol{r}^{(1)}, \boldsymbol{r}^{(2)}\right)\right]^{2} /\left[\rho(\boldsymbol{r}) \rho\left(\boldsymbol{r}^{(1)}\right) \rho\left(\boldsymbol{r}^{(2)}\right)\right],
$$

which satisfy the relevant consecutive bridge normalizations:

$$
\begin{aligned}
\int d \boldsymbol{r}^{(1)} \int d \boldsymbol{r}^{(2)}\left[\int d \boldsymbol{r}^{\prime} P\left(\boldsymbol{r} \rightarrow \boldsymbol{r}^{\prime} ; \boldsymbol{r}^{(1)}, \boldsymbol{r}^{(2)}\right)\right] & =\int d \boldsymbol{r}^{(1)}\left[\int d \boldsymbol{r}^{(2)} \int P\left(\boldsymbol{r} \rightarrow \boldsymbol{r}^{(2)} ; \boldsymbol{r}^{(1)}\right)\right] \\
& =\int d \boldsymbol{r}^{(1)} P\left(\boldsymbol{r}^{(1)} \mid \boldsymbol{r}\right)=1 .
\end{aligned}
$$

It thus follows from Eqs. [(30), (34)] and [(37), (39)] that the resultant phase exhibited by the amplitude of the signal bridge-propagation is given by the sum of the relative phases (phase-shifts) in each of its consecutive direct stages [see Eq. (29)].

Consider a single particle $(N=1)$ occupying the (bonding) $\mathrm{MO} \varphi_{b}(\boldsymbol{r}, \sigma)=$ $\phi_{b}(\boldsymbol{r}) \xi(\sigma)$. This illustrative example gives:

$$
\begin{array}{ll}
\gamma\left(\boldsymbol{r}, \boldsymbol{r}^{\prime}\right)=\Gamma_{\boldsymbol{r}, \boldsymbol{r}^{\prime}}=\phi_{b}(\boldsymbol{r}) \phi_{b}^{*}\left(\boldsymbol{r}^{\prime}\right), & \rho(\boldsymbol{r})=p(\boldsymbol{r})=\gamma(\boldsymbol{r}, \boldsymbol{r})=\left|\phi_{b}(\boldsymbol{r})\right|^{2}, \\
A\left(\boldsymbol{r}^{\prime} \mid \boldsymbol{r}\right)=\phi_{b}(\boldsymbol{r}) \phi_{b}^{*}\left(\boldsymbol{r}^{\prime}\right) / p(\boldsymbol{r})^{1 / 2}, & \pi\left(\boldsymbol{r}, \boldsymbol{r}^{\prime}\right)=\Omega\left(\boldsymbol{r}, \boldsymbol{r}^{\prime}\right)=p(\boldsymbol{r}) p\left(r^{\prime}\right), \quad P\left(\boldsymbol{r}^{\prime} \mid \boldsymbol{r}\right)=p\left(\boldsymbol{r}^{\prime}\right) .
\end{array}
$$


In this simplest case the two locations are independent of each other, thus giving rise to the factorized joint probability of the two position arguments in $\pi\left(\boldsymbol{r}, \boldsymbol{r}^{\prime}\right)$. It should be emphasized that all these distributions are single-electron in character, with both locations referring to the admissible positions of an electron in the molecular bond system.

\section{Entropic descriptors of local communications}

Consider now the overall entropy/information descriptors [3,4,7,8,11-13] of a transmission of the electron-assignment "signals" in the local information channel of a molecule, originating from its bond system detrmined by the occupied SMO subspace $\boldsymbol{\varphi}$. In this "device" the signal emitted from the continuous "inputs" $\boldsymbol{r} \in \mathfrak{R}$ of the channel "source" is propagated via the SMO into the continuous "outputs" $r$ ' $\in \mathfrak{R}$ ' of the channel "receiver". The network of communications in the classical (probability) channel,

$$
\left\{p(\boldsymbol{r}) \rightarrow \boldsymbol{r} \rightarrow P\left(\boldsymbol{r}^{\prime} \mid \boldsymbol{r}\right) \rightarrow \boldsymbol{r}^{\prime} \rightarrow p^{\prime}\left(\boldsymbol{r}^{\prime}\right)\right\}
$$

is determined by the conditional probabilities $\left\{P\left(\boldsymbol{r}^{\prime} \mid \boldsymbol{r}\right)\right\}$, while the amplitudes $A\left(\boldsymbol{r}^{\prime} \mid \boldsymbol{r}\right)$ determine the associated quantum (amplitude) scattering system:

$$
\left\{A(\boldsymbol{r}) \equiv[\rho(\boldsymbol{r})]^{1 / 2} \rightarrow|\boldsymbol{r}\rangle \rightarrow A\left(\boldsymbol{r}^{\prime} \mid \boldsymbol{r}\right) \rightarrow\left|\boldsymbol{r}^{\prime}\right\rangle \rightarrow \int d \boldsymbol{r} \gamma\left(\boldsymbol{r}, \boldsymbol{r}^{\prime}\right) \equiv A\left(\boldsymbol{r}^{\prime}\right)\right\}
$$

We again emphasize that the interference of this amplitude system gives rise to the resultant phases of the admissible cascade channels, which conserve the stationary molecular communications in the multi-stage scatterings involving intermediate particle locations.

In Eq. (44) the molecular amplitude $A(\boldsymbol{r}) \equiv[\rho(\boldsymbol{r})]^{1 / 2}$ shapes the input-signal of the quantum channel [see also Eq. (18)], which characterizes how this communication system is used in the ground-state of the molecule. The resultant output-amplitude $A\left(\boldsymbol{r}^{\prime}\right)$ has the following geometrical interpretation [see Eq. (16)]: this continuous "summation" ("contraction") of $\gamma\left(\boldsymbol{r}, \boldsymbol{r}^{\prime}\right)$ over the input events $|\Re\rangle=\{|\boldsymbol{r}\rangle\}$ in the molecular (one-electron) Hilbert space,

$$
A\left(\boldsymbol{r}^{\prime}\right)=\int d \boldsymbol{r} \gamma\left(\boldsymbol{r}, \boldsymbol{r}^{\prime}\right)=\int d \boldsymbol{r}\left(\langle\boldsymbol{r}| \hat{\mathrm{P}}_{\varphi}\right)\left(\hat{\mathrm{P}}_{\varphi}\left|\boldsymbol{r}^{\prime}\right\rangle\right) \equiv\left\langle\Re^{b} \mid \boldsymbol{r}^{\prime b}\right\rangle,
$$

represents the scalar product between the ( $N$-dimensional) bond-subspace projection of the infinitely-dimensional (continuous) vector space spanned by the position basisset vectors, $\left|\Re^{b}\right\rangle=\left\{\left|\boldsymbol{r}^{b}\right\rangle=\hat{\mathrm{P}}_{\varphi}|\boldsymbol{r}\rangle=\sum_{s}\left|\phi_{s}\right\rangle \phi_{s}^{*}(\boldsymbol{r})\right\} \equiv \hat{\mathrm{P}}_{\varphi}|\Re\rangle$, and the corresponding projection of the specified output state-vector, $\left|\boldsymbol{r}^{\prime b}\right\rangle=\hat{\mathrm{P}}_{\varphi}\left|\boldsymbol{r}^{\prime}\right\rangle$. In other words, this resultant output amplitude amounts to the $N$-dimensional projection of $\left|\boldsymbol{r}^{\prime b}\right\rangle$ onto $\left|\Re^{b}\right\rangle$.

Therefore, in determining the purely molecular descriptors of the local channels the input signal in the classical and quantum local channels is characterized by the molecular probability distribution $p(\boldsymbol{r})$ and the associated amplitude $A(\boldsymbol{r})$, respectively. 


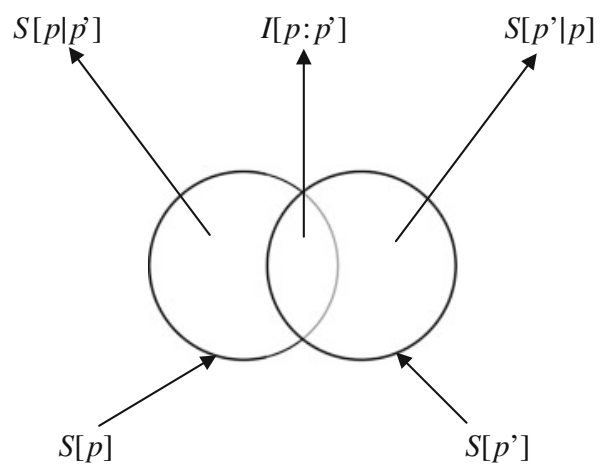

Fig. 3 Qualitative diagram of the conditional-entropy and mutual-information quantities for two spatial probability distributions $p(\boldsymbol{r})$ and $p^{\prime}\left(\boldsymbol{r}^{\prime}\right)$ of Eq. 43. Two circles enclose areas representing the entropies $S[p]$ and $S\left[p^{\prime}\right]$ of the molecular input and output probability densities, respectively, while their common (overlap) area corresponds to the mutual information $I\left[p: p^{\prime}\right]$ in these two distributions. The remaining part of each circle represents the corresponding conditional entropy, $S\left[p \mid p^{\prime}\right]$ or $S\left[p^{\prime} \mid p\right]$, measuring the residual uncertainty about events in one set of local events, when one has the full knowledge of the occurrence of events in the other set of local outcomes. The area enclosed by the envelope of two circles then represents the entropy of the "product" (joint) distribution: $S[\pi]=S[p]+S\left[p^{\prime}\right]-I\left[p: p^{\prime}\right]=S[p]+S\left[p^{\prime} \mid p\right]=$ $S\left[p^{\prime}\right]+S\left[p \mid p^{\prime}\right]$

The transmission of signals in the communication channel is randomly disturbed thus exhibiting a communication noise. The classical signal propagation is described by the conditional-probability kernel $P\left(\boldsymbol{r}^{\prime} \mid \boldsymbol{r}\right)$ of the local-outputs given local-inputs, while the associated amplitude kernel $A\left(\boldsymbol{r}^{\prime} \mid \boldsymbol{r}\right)$ determines the amplitude propagation. The output signal $p^{\prime}\left(\boldsymbol{r}^{\prime}\right)$ then conserves the input distribution [see Eq. (21)]:

$$
p^{\prime}\left(\boldsymbol{r}^{\prime}\right)=\int p(\boldsymbol{r}) P\left(\boldsymbol{r}^{\prime} \mid \boldsymbol{r}\right) d \boldsymbol{r}=\int p(\boldsymbol{r})\left[\pi\left(\boldsymbol{r}, \boldsymbol{r}^{\prime}\right) / p(\boldsymbol{r})\right] d \boldsymbol{r}=\int \pi\left(\boldsymbol{r}, \boldsymbol{r}^{\prime}\right) d \boldsymbol{r}=p\left(\boldsymbol{r}^{\prime}\right)
$$

The Shannon entropy of the joint ("product") distribution $\pi\left(\boldsymbol{r}, \boldsymbol{r}^{\prime}\right)$ can be expressed as the sum of the average entropy in the "marginal" (input) probability distribution $p(r)$,

$$
S[p]=-\int p(\boldsymbol{r}) \log p(\boldsymbol{r}) d \boldsymbol{r}
$$

and the average conditional entropy in the local-outputs given local-inputs (see Fig. 3),

$$
\begin{aligned}
S\left[p^{\prime} \mid p\right] & =-\iint \pi\left(\boldsymbol{r}, \boldsymbol{r}^{\prime}\right) \log P\left(\boldsymbol{r}^{\prime} \mid \boldsymbol{r}\right) d \boldsymbol{r} d \boldsymbol{r}^{\prime}=-\int p(\boldsymbol{r})\left[\int P\left(\boldsymbol{r}^{\prime} \mid \boldsymbol{r}\right) \log P\left(\boldsymbol{r}^{\prime} \mid \boldsymbol{r}\right) d \boldsymbol{r}^{\prime}\right] d \boldsymbol{r}, \\
S[\pi] & =-\iint \pi\left(\boldsymbol{r}, \boldsymbol{r}^{\prime}\right) \log \pi\left(\boldsymbol{r}, \boldsymbol{r}^{\prime}\right) d \boldsymbol{r} d \boldsymbol{r}^{\prime}=-\iint p(\boldsymbol{r}) P\left(\boldsymbol{r}^{\prime} \mid \boldsymbol{r}\right)\left[\log p(\boldsymbol{r})+\log P\left(\boldsymbol{r}^{\prime} \mid \boldsymbol{r}\right)\right] d \boldsymbol{r} d \boldsymbol{r}^{\prime} \\
& =-\int p(\boldsymbol{r}) \log p(\boldsymbol{r}) d \boldsymbol{r}\left[\int P\left(\boldsymbol{r}^{\prime} \mid \boldsymbol{r}\right) d \boldsymbol{r}^{\prime}\right]-\int p(\boldsymbol{r})\left[\int P\left(\boldsymbol{r}^{\prime} \mid \boldsymbol{r}\right) \log P\left(\boldsymbol{r}^{\prime} \mid \boldsymbol{r}\right) d \boldsymbol{r}^{\prime}\right] d \boldsymbol{r} \\
& \equiv S[p]+S\left[p^{\prime} \mid p\right]
\end{aligned}
$$

The conditional entropy $S\left[p^{\prime} \mid p\right]$ represents the extra amount of the information about the occurrence of the output local events, given that the local input events are known to have already occurred. In other words: the amount of entropy/information $S[\pi]$ in the joint probability density of simultaneously observing the input-output localization 
events $\left\{\left(\boldsymbol{r}, \boldsymbol{r}^{\prime}\right) \equiv\left(\boldsymbol{r} \wedge \boldsymbol{r}^{\prime}\right)\right\}$, the arguments of the two continuous molecular probability distributions $p$ and $p^{\prime}$, respectively, i.e., of the the emitted and received signals in the molecular ground-state channel, equals to the amount of information $S[p]$ received by observing the input events $\{\boldsymbol{r}\}$, supplemented by the extra information $S\left[p^{\prime} \mid p\right]$ provided by the occurrence of the output events $\left\{\boldsymbol{r}^{\prime}\right\}$, when $\{\boldsymbol{r}\}$ are known to have occurred already.

The common amount of the information in two dependent local events $\boldsymbol{r}$ and $\boldsymbol{r}^{\prime}$, $I\left(\boldsymbol{r}: \boldsymbol{r}^{\prime}\right)$, measuring the information about $\boldsymbol{r}$ provided by the occurrence of $\boldsymbol{r}^{\prime}$, determines the mutual information in these two local events:

$$
\begin{aligned}
I\left(\boldsymbol{r}: \boldsymbol{r}^{\prime}\right) & =\log \left[\pi\left(\boldsymbol{r}, \boldsymbol{r}^{\prime}\right) /\left[p(\boldsymbol{r}) p^{\prime}\left(\boldsymbol{r}^{\prime}\right)\right]=\log \left[P\left(\boldsymbol{r} \mid \boldsymbol{r}^{\prime}\right) / p(\boldsymbol{r})\right]\right. \\
& \equiv \log \left[P\left(\boldsymbol{r}^{\prime} \mid \boldsymbol{r}\right) / p^{\prime}\left(\boldsymbol{r}^{\prime}\right)\right]=I\left(\boldsymbol{r}^{\prime}: \boldsymbol{r}\right) .
\end{aligned}
$$

It vanishes, when both events are independent, $\pi\left(\boldsymbol{r}, \boldsymbol{r}^{\prime}\right)=\pi^{i n d .}\left(\boldsymbol{r}, \boldsymbol{r}^{\prime}\right) \equiv p(\boldsymbol{r}) p^{\prime}\left(\boldsymbol{r}^{\prime}\right)$, i.e., when the occurrence of one event does not influence (or condition) the probability of the occurrence of the other event, and it is negative, when the occurrence of one event makes a non-occurrence of the other event more likely. It also follows from the preceding equation that

$$
I\left(\boldsymbol{r}: \boldsymbol{r}^{\prime}\right)=I(\boldsymbol{r})-I\left(\boldsymbol{r} \mid \boldsymbol{r}^{\prime}\right)=I\left(\boldsymbol{r}^{\prime}\right)-I\left(\boldsymbol{r}^{\prime} \mid \boldsymbol{r}\right)=I(\boldsymbol{r})+I\left(\boldsymbol{r}^{\prime}\right)-I\left(\boldsymbol{r}, \boldsymbol{r}^{\prime}\right),
$$

where the self-information of the joint two-point event $I\left(\boldsymbol{r}, \boldsymbol{r}^{\prime}\right)=-\log \pi\left(\boldsymbol{r}, \boldsymbol{r}^{\prime}\right), I(\boldsymbol{r})=$ $-\log p(\boldsymbol{r}), I\left(\boldsymbol{r} \mid \boldsymbol{r}^{\prime}\right)=-\log P\left(\boldsymbol{r} \mid \boldsymbol{r}^{\prime}\right)$, etc.

Thus, the information in the joint occurrence of two local input and output events $r$ and $\boldsymbol{r}^{\prime}$, respectively, is the information in the occurrence of $\boldsymbol{r}$ plus that in the occurrence of $\boldsymbol{r}^{\prime}$ minus the mutual information. Clearly, for independent events, when $\pi\left(\boldsymbol{r}, \boldsymbol{r}^{\prime}\right)=$ $\pi^{\text {ind. }}\left(\boldsymbol{r}, \boldsymbol{r}^{\prime}\right)$ and hence $I\left(\boldsymbol{r}: \boldsymbol{r}^{\prime}\right)=0, I^{\text {ind. }}\left(\boldsymbol{r}, \boldsymbol{r}^{\prime}\right)=I(\boldsymbol{r})+I\left(\boldsymbol{r}^{\prime}\right)$.

The mutual information of the local event with itself defines its self-information:

$$
I(\boldsymbol{r}: \boldsymbol{r}) \equiv I(\boldsymbol{r})=\log [P(\boldsymbol{r} \mid \boldsymbol{r}) / p(\boldsymbol{r})]=-\log p(\boldsymbol{r}),
$$

since $P(\boldsymbol{r} \mid \boldsymbol{r})=1$. It vanishes, when $p(\boldsymbol{r})=1$, i.e., when there is no uncertainty about the occurrence of $\boldsymbol{r}$, so that the occurrence of this event removes no uncertainty, hence conveys no information. This quantity provides a measure of the uncertainty about the occurrence of the event itself, i.e., the information received when this event actually occurs. The Shannon entropy of Eq. (47) can be thus interpreted as the mean value of self-information densities in individual events, $\{I(\boldsymbol{r})=-\log p(\boldsymbol{r})\}, S[p]=$ $\int p(\boldsymbol{r}) I(\boldsymbol{r}) d \boldsymbol{r}$. One similarly defines the average mutual information in two probability distributions (see Fig. 3) as the mean value of the mutual information quantities for individual joint-localization events:

$$
\begin{aligned}
I\left[p: p^{\prime}\right] & =\iint \pi\left(\boldsymbol{r}, \boldsymbol{r}^{\prime}\right) I\left(\boldsymbol{r}: \boldsymbol{r}^{\prime}\right) d \boldsymbol{r} d \boldsymbol{r}^{\prime}=\iint \pi\left(\boldsymbol{r}, \boldsymbol{r}^{\prime}\right) \log \left[\pi\left(\boldsymbol{r}, \boldsymbol{r}^{\prime}\right) / \pi^{i n d} .\left(\boldsymbol{r}, \boldsymbol{r}^{\prime}\right)\right] d \boldsymbol{r} d \boldsymbol{r}^{\prime} \\
& =S[p]+S\left[p^{\prime}\right]-S[\pi]=S[p]-S\left[p \mid p^{\prime}\right]=S\left[p^{\prime}\right]-S\left[p^{\prime} \mid p\right] \geq 0,
\end{aligned}
$$

where the equality holds only for the independent input and output distributions. Indeed, the amount of uncertainty in $p^{\prime}$ can only decrease, when $p$ has been known 
beforehand, $S\left[p^{\prime}\right] \geq S\left[p^{\prime} \mid p\right]=S\left[p^{\prime}\right]-I\left[p: p^{\prime}\right]$, with equality being observed only when the two sets of events are independent, thus giving non-overlapping entropy circles in Fig. 3.

The average mutual information is an example of the entropy deficiency (cross entropy, missing information, information distance, directed divergence) of Kullback and Leibler [5,6]. Indeed, for the continuous joint-probability scheme identified by the dependent events $\left\{\left(\boldsymbol{r}, \boldsymbol{r}^{\prime}\right)\right\}$ and their probabilities $\pi\left(\boldsymbol{r}, \boldsymbol{r}^{\prime}\right)$, this discrimination information in $\pi$ with respect to the reference distribution of independent joint events $\pi^{i n d .}\left(\boldsymbol{r}, \boldsymbol{r}^{\prime}\right)$ is defined by the functional of Eq. $(52): \Delta S\left[\pi \mid \pi^{i n d \cdot}\right]=I\left[p: p^{\prime}\right] \geq 0$. This quantity provides a measure of the information-distance between the two distributions compared, and hence also the criterion of their information resemblance. The more the two joint distributions differ from one another, the larger the information distance. Notice that $\Delta S\left[\pi \mid \pi^{i n d .}\right]=0$ marks the vanishing surprisals in all joint events, i.e., when the two probability distributions are identical. The average mutual information thus reflects a degree of a dependence between events defining the dependent and independent probability densities. A similar informationdistance interpretation can be attributed to the average conditional entropy of Eq. (48): $S\left[p^{\prime} \mid p\right]=S\left[p^{\prime}\right]-\Delta S\left[\pi \mid \pi^{i n d} \cdot\right]$.

The average conditional entropy descriptor of the local probability channel is thus given by the following functional of $\gamma\left(\boldsymbol{r}, \boldsymbol{r}^{\prime}\right)$ for the molecular ground-state configuration:

$$
\begin{aligned}
S\left[p^{\prime} \mid p\right] & =-\iint P\left(\boldsymbol{r}, \boldsymbol{r}^{\prime}\right) \log P\left(\boldsymbol{r}^{\prime} \mid \boldsymbol{r}\right) d \boldsymbol{r} d \boldsymbol{r}^{\prime}=-\iint P_{\boldsymbol{r}, \boldsymbol{r}^{\prime}}[\gamma] \log P_{\boldsymbol{r} \rightarrow \boldsymbol{r}^{\prime}}[\gamma] d \boldsymbol{r} d \boldsymbol{r}^{\prime} \equiv S_{\Re \rightarrow \Re^{\prime}}[\gamma] \\
& =-N^{-1} \iint \gamma\left(\boldsymbol{r}, \boldsymbol{r}^{\prime}\right) \gamma\left(\boldsymbol{r}^{\prime}, \boldsymbol{r}\right)\left\{\log \left[\gamma\left(\boldsymbol{r}, \boldsymbol{r}^{\prime}\right) \gamma\left(\boldsymbol{r}, \boldsymbol{r}^{\prime}\right)\right]-\log \gamma(\boldsymbol{r}, \boldsymbol{r})\right\} d \boldsymbol{r}^{\prime} d \boldsymbol{r} \\
& \equiv-N^{-1} \iint \Omega\left(\boldsymbol{r}, \boldsymbol{r}^{\prime}\right) \log \Omega\left(\boldsymbol{r}, \boldsymbol{r}^{\prime}\right) d \boldsymbol{r}^{\prime} d \boldsymbol{r}+N^{-1} \int\left[\int \Omega\left(\boldsymbol{r}, \boldsymbol{r}^{\prime}\right) d \boldsymbol{r}^{\prime}\right] \log \rho(\boldsymbol{r}) d \boldsymbol{r} \\
& =N^{-1}(S[\Omega]-S[\rho]) .
\end{aligned}
$$

It thus reflects the difference between the Shannon-entropy

$$
S[\Omega]=-\iint \Omega\left(\boldsymbol{r}, \boldsymbol{r}^{\prime}\right) \log \Omega\left(\boldsymbol{r}, \boldsymbol{r}^{\prime}\right) d \boldsymbol{r} d \boldsymbol{r}^{\prime}
$$

of the two-point distribution $\Omega\left(\boldsymbol{r}, \boldsymbol{r}^{\prime}\right)=\left|\Gamma_{\boldsymbol{r}, \boldsymbol{r}^{\prime}}\right|^{2}$,

$$
\Omega\left(\boldsymbol{r}, \boldsymbol{r}^{\prime}\right)=\Omega_{\boldsymbol{r}, \boldsymbol{r}^{\prime}}[\gamma]=\gamma\left(\boldsymbol{r}, \boldsymbol{r}^{\prime}\right) \gamma\left(\boldsymbol{r}^{\prime}, \boldsymbol{r}\right)=N P\left(\boldsymbol{r}, \boldsymbol{r}^{\prime}\right),
$$

and the entropy of the electron density $\rho(\boldsymbol{r})=N p(\boldsymbol{r})$,

$$
S[\rho]=-\int \rho(\boldsymbol{r}) \log \rho(\boldsymbol{r}) d \boldsymbol{r}=-N \int p(\boldsymbol{r})[\log N+\log p(\boldsymbol{r})] d \boldsymbol{r}=-N \log N+N S[p] .
$$

The electron distribution of the system promolecule $[11,34]$ is described by the the sum of the molecularly placed electron densities $\left\{\rho_{\mathrm{X}}{ }^{0}(\boldsymbol{r})\right\}$ of the free constituent atoms,

$$
\rho^{0}(\boldsymbol{r})=N p^{0}(\boldsymbol{r})=\sum_{\mathrm{X}} \rho_{\mathrm{X}}^{0}(\boldsymbol{r})
$$


determining the diagonal part $\rho^{0}(\boldsymbol{r})=\gamma^{0}(\boldsymbol{r}, \boldsymbol{r})$ of the promolecular density matrix

$$
\gamma^{0}\left(\boldsymbol{r}, \boldsymbol{r}^{\prime}\right)=\sum_{\mathrm{X}} \gamma_{\mathrm{X}}^{0}\left(\boldsymbol{r}, \boldsymbol{r}^{\prime}\right)
$$

The shape factor $p^{0}(\boldsymbol{r})$ provides the reference input signal for establishing the corresponding "difference" descriptor of the information-flow in the local channel, given by the mutual information:

$$
\begin{aligned}
& I\left[p^{0}: p\right]=\int\left[\int P\left(\boldsymbol{r}, \boldsymbol{r}^{\prime}\right)\right] \log \left[P\left(\boldsymbol{r}^{\prime} \mid \boldsymbol{r}\right) / p^{0}(\boldsymbol{r})\right] d \boldsymbol{r}^{\prime} d \boldsymbol{r} \equiv I_{\Re \rightarrow \Re^{\prime}}\left[\gamma, \gamma^{0}\right] \\
& \left.=-S_{\Re \rightarrow \Re^{\prime}}[\gamma]-N^{-1} \int\left[\int \Omega\left(\boldsymbol{r}, \boldsymbol{r}^{\prime}\right) d \boldsymbol{r}^{\prime}\right] \log p^{0}(\boldsymbol{r})\right\} d \boldsymbol{r} \\
& \equiv-S_{\Re \rightarrow \Re^{\prime}}[\gamma]+S[p]+\Delta S\left[p \mid p^{0}\right] \text {, }
\end{aligned}
$$

where the entropy deficiency in the molecular (ground-state) probability $p$, relative to the promolecular distribution $p^{0}$,

$$
\Delta S\left[p \mid p^{0}\right]=\int p(\boldsymbol{r}) \log \left[p(\boldsymbol{r}) / p^{0}(\boldsymbol{r})\right] d \boldsymbol{r} \equiv \int p(\boldsymbol{r}) I(\boldsymbol{r}) d \boldsymbol{r},
$$

measures the average value of the local "surprisal" $I(\boldsymbol{r})$, which reflects the local information distance between the two distributions. Since $p(\boldsymbol{r})$ and $p^{0}(\boldsymbol{r})$ strongly resemble one another, being distinguished only by relatively minor displacements in the AIM valence shells, $\Delta S\left[p \mid p^{0}\right]$ generally constitutes a relatively minor contribution. This similarity index exactly disappears for the molecular input signal $p(\boldsymbol{r}), \Delta S[p \mid p]=0$, thus giving a modified value of the average mutual information of the local channel:

$$
I\left[p: p^{\prime}\right]=-S_{\Re \rightarrow \Re^{\prime}}[\gamma]+S[p[\gamma]] \equiv I_{\mathfrak{R} \rightarrow \Re^{\prime}}[\gamma]
$$

The corresponding overall bond multiplicity indices [11-13], combining the relevant conditional entropy and mutual information descriptors, thus read:

$$
\begin{aligned}
M\left[p^{0} ; p\right] & =S\left[p^{\prime} \mid p\right]+I\left[p^{0}: p\right]=S[p]+\Delta S\left[p \mid p^{0}\right] \\
M\left[p ; p^{\prime}\right] & =S\left[p^{\prime} \mid p\right]+I\left[p: p^{\prime}\right]=S[p] .
\end{aligned}
$$

Therefore, the purely molecular overall IT bond multiplicity, of the molecular probability channel probed by the molecular input signal, recovers the Shannon entropy of the ground-state probability of electrons. The difference index

$$
\Delta M \equiv M\left[p^{0} ; p\right]-M\left[p ; p^{\prime}\right]=\Delta S\left[p \mid p^{0}\right]
$$

reflects the overall similarity between the molecular probability distribution $p$ and the promolecular reference $p^{0}$. It disappears in the separated atoms limit (SAL), where the two distributions become identical. 
As an illustration, let us again consider the simplest case invoked at the end of Sect. 3, of a single electron $(N=1)$ occupying the lowest SMO, $\varphi(\boldsymbol{r}, \sigma)=\phi(\boldsymbol{r}) \xi(\sigma)$. The relevant electron distributions [see Eq. (42)] then read:

$$
\begin{aligned}
& \gamma\left(\boldsymbol{r}, \boldsymbol{r}^{\prime}\right)=\Gamma_{\boldsymbol{r}, \boldsymbol{r}^{\prime}}=\phi(\boldsymbol{r}) \phi^{*}\left(\boldsymbol{r}^{\prime}\right), p(\boldsymbol{r})=\gamma(\boldsymbol{r}, \boldsymbol{r})=|\phi(\boldsymbol{r})|^{2}, \quad A\left(\boldsymbol{r}^{\prime} \mid \boldsymbol{r}\right)=\phi(\boldsymbol{r}) \phi^{*}\left(\boldsymbol{r}^{\prime}\right) /[p(\boldsymbol{r})]^{1 / 2}, \\
& \pi\left(\boldsymbol{r}, \boldsymbol{r}^{\prime}\right)=\Omega\left(\boldsymbol{r}, \boldsymbol{r}^{\prime}\right)=p(\boldsymbol{r}) p\left(\boldsymbol{r}^{\prime}\right), P\left(\boldsymbol{r}^{\prime} \mid \boldsymbol{r}\right)=p\left(\boldsymbol{r}^{\prime}\right) .
\end{aligned}
$$

The two locations are independent of each other, thus giving rise to the factorized form of $\pi\left(\boldsymbol{r}, \boldsymbol{r}^{\prime}\right)$ and, hence, to the vanishing mutual information $I\left[p: p^{\prime}\right]=0$.

In what follows we examine the two model systems: the hydrogen-like atom $\mathrm{H}(Z), \phi(r)=1 s(r)=\left(Z^{3} / \pi\right)^{1 / 2} \exp (-Z r)$, and the model "half"-bond in $\mathrm{H}_{2}^{+}$, calculated for the equilibrium bond length in $\mathrm{H}_{2}, R_{e}\left[\mathrm{H}_{2}\right]=1.4$ a.u., when

$$
\phi(\boldsymbol{r})=\phi_{b}(\boldsymbol{r})=N_{b}\left[1 s_{\mathrm{A}}(\boldsymbol{r})+1 s_{\mathrm{B}}(\boldsymbol{r})\right] .
$$

Here, the normalization constant for this internuclear distance $N_{b}=0.534$.

In the simplest atomic system $\mathrm{H}(Z)$ the relevant average noise (conditional entropy) can be calculated analytically. The relevant distributions of Eq. (64),

$$
\begin{aligned}
& \gamma\left(\boldsymbol{r}, \boldsymbol{r}^{\prime}\right)=\left(Z^{3} / \pi\right) \exp \left[-Z\left(\boldsymbol{r}+\boldsymbol{r}^{\prime}\right)\right], p(\boldsymbol{r})=\left(Z^{3} / \pi\right) \exp (-2 Z r), \\
& \pi\left(\boldsymbol{r}, \boldsymbol{r}^{\prime}\right)=\Omega\left(\boldsymbol{r}, \boldsymbol{r}^{\prime}\right)=p(\boldsymbol{r}) p\left(\boldsymbol{r}^{\prime}\right), P\left(\boldsymbol{r}^{\prime} \mid \boldsymbol{r}\right)=p\left(\boldsymbol{r}^{\prime}\right),
\end{aligned}
$$

give for the molecular input and output distributions, $p^{\prime}=p$,

$$
\begin{aligned}
S\left[p^{\prime} \mid p\right] & =-\iint p(\boldsymbol{r}) p\left(\boldsymbol{r}^{\prime}\right) \log p\left(\boldsymbol{r}^{\prime}\right) d \boldsymbol{r} d \boldsymbol{r}^{\prime}=\left[\int p(\boldsymbol{r}) d \boldsymbol{r}\right]\left[-\int p\left(\boldsymbol{r}^{\prime}\right) \log p\left(\boldsymbol{r}^{\prime}\right) d \boldsymbol{r}^{\prime}\right] \\
& =S[p]=3-\ln \left(Z^{3} / \pi\right)[\text { nats }] .
\end{aligned}
$$

For the hydrogen atom $(Z=1)$ it predicts

$$
S[p]=3+\ln \pi=6.1 \text { nats }=8.8 \text { bits. }
$$

The entropy of Eq. (67) correctly predicts a decreasing noise content in local communications with increasing nuclear charge of the hydrogen-like atom, which exactly vanishes for the critical value

$$
Z^{*}=\exp [(3+\ln \pi) / 3]=7.75 \text { a.u. }
$$

In examining $\mathrm{H}_{2}^{+}\left[R_{\mathrm{AB}}=1.4\right.$ a.u. $]$ one uses the numerically estimated [11,25] value of the Shannon entropy for $\mathrm{H}_{2}\left[R_{\mathrm{AB}}=1.4 \mathrm{a}\right.$.u. $], S[\rho]=6.6$ bits. This gives the associated entropy $S[p]$ of the probability (shape factor) [see Eq. (56)],

$$
S[p]=1 / 2\left\{S[\rho]+2 \log _{2} 2\right\}=4.3 \text { bits, }
$$

which also marks the conditional entropy in $\mathrm{H}_{2}^{+}\left[R_{\mathrm{AB}}=1.4\right.$ a.u. $]$. Since the entropy of Eq. (68) also determines the noise level of the corresponding promolecule, one 


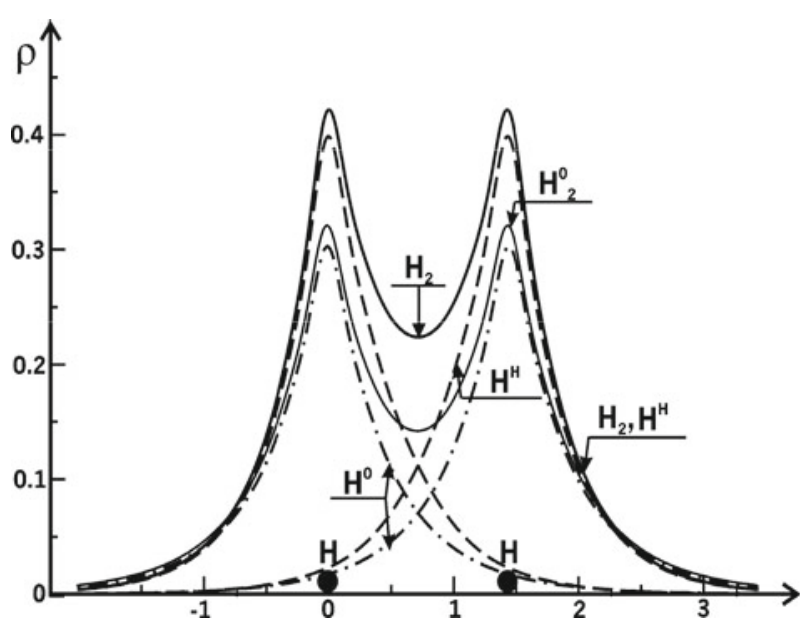

Fig. 4 The Hirshfeld electron densities of bonded hydrogen atoms $\left(\mathrm{H}^{\mathrm{H}}\right)$ obtained from the molecular density $\left(\mathrm{H}_{2}\right)$. The free-hydrogen densities $\left(\mathrm{H}^{0}\right)$ and the resulting electron density of the promolecule $\left(\mathrm{H}_{2}^{0}\right)$ are also shown for comparison. The density and inter-nuclear distance are in a.u. The zero cusps at nuclear positions are the artifacts of the Gaussian basis set used in DFT calculations [11,25]

thus concludes that this prototype (covalent) half-bond marks a decisive lovering of the communication noise, by about 4.5 bits, compared to the free-hydrogen entropy in this artificially shortened bond of $\mathrm{H}_{2}^{+}$. It is expected to be much lower at the equilibrium distance $R_{e}\left[\mathrm{H}_{2}^{+}\right]=2.0$ a.u., since then the perturbing influence of the bond partner should be less felt by both AIM.

Indeed, the increased "order", localization influence in the molecule is due to an effective contraction of the AIM distributions (see Fig. 4), relative to the free-atom density, due to the presence of the unshielded nucleus of the other atom. A similar effect has been observed in $\mathrm{H}_{2}[11,25]$, where $S\left[\rho^{0}\right]=7.4$ bits or $S\left[p^{0}\right]=4.7$ bits, thus again predicting a slight lowering, by about 0.4 bits, of the average conditional entropy in the molecule $S[p]=1 / 2\left\{S[\rho]+2 \log _{2} 2\right\}=4.3$ bits, $S[p]=4.3$ bits, relative to its promolecular reference $S\left[p^{0}\right]=4.7$ bits, despite the opposite effect of the electron delocalization via the covalent chemical bond.

\section{Conclusion}

Information Theory provides an attractive perspective on the molecular equilibria, multiplicity, composition/localization of chemical bonds and their information origins. We have presented in this analysis some new developments in the local information systems within the single-configuration CTCB, derived using the (bond-projected) superposition principle of quantum mechanics. The probability amplitude of the joint, two-point events in the molecular bond system, defined by the configuration occupied SMO, is then given by the one-electron density matrix. These joint-probabilities define the associated conditional- probabilities, which determine the system classical communication links. The interference and phase relations in the sequential cas- 
cades of the molecular amplitudes have been examined. The conditional-entropy and mutual-information descriptors of such local networks, measuring the channel average communication "noise" and information flow, respectively, have been linked to the chemical covalency and ionicity characteristics.

This local communication theory (LCT) introduces the classical (probability) and quantum (amplitude) channels of the direct and indirect communications between infinitesimal volume elements in molecules, and links their entropic descriptors to generalized IT bond multiplicities and their covalent/ionic components. This development can be straightforwardly generalized into the multi-determinantal (CI) description using eithr the CI ensembles of the occupied SMO [80,81] or the natural orbital (NO) framework [44].

Open Access This article is distributed under the terms of the Creative Commons Attribution License which permits any use, distribution, and reproduction in any medium, provided the original author(s) and the source are credited.

\section{References}

1. R.A. Fisher, Proc. Camb. Phil. Soc. 22, 700 (1925)

2. B.R. Frieden, Physics from the Fisher Information: A Unification, 2nd edn. (Cambridge University Press, Cambridge, 2004)

3. C.E. Shannon, Bell Syst. Technol. J. 27, 379, 623 (1948)

4. C.E. Shannon, W. Weaver, The Mathematical Theory of Communication (University of Illinois, Urbana, 1949)

5. S. Kullback, R.A. Leibler, Ann. Math. Stat. 22, 79 (1951)

6. S. Kullback, Information Theory and Statistics (Wiley, New York, 1959)

7. N. Abramson, Information Theory and Coding (McGraw-Hill, New York, 1963)

8. P.E. Pfeifer, Concepts of Probability Theory, 2nd edn. (Dover, New York, 1978)

9. R.F. Nalewajski, J. Math. Chem. 51, 297 (2013)

10. R.F. Nalewajski, Entropic concepts in electronic structure theory. Found. Chem. doi:10.1007/ s10698-012-9168-7

11. R.F. Nalewajski, Information Theory of Molecular Systems (Elsevier, Amsterdam, 2006)

12. R.F. Nalewajski, Information Origins of the Chemical Bond (Nova, New York, 2010)

13. R.F. Nalewajski, Perspectives in Electronic Structure Theory (Springer, Heidelberg, 2012)

14. R.F. Nalewajski, in Mathematical Chemistry, ed. by W.I. Hong (Nova Science Publishers, New York, 2011), pp. 247-325

15. R.F. Nalewajski, in Chemical Information and Computation Challenges in Twenty-First Century, ed. by M.V. Putz (Nova Science Publishers, New York, 2012), pp. 61-100

16. R.F. Nalewajski, P. de Silva, J. Mrozek, in Theoretical and Computational Developments in Modern Density Functional Theory, ed. by A.K. Roy (Nova, New York, 2012), pp. 561-588

17. R.F. Nalewajski, in Frontiers in Modern Theoretical Chemistry: Concepts and Methods (Dedicated to B. M. Deb), ed. by P.K. Chattaraj, S.K. Ghosh (Taylor \& Francis/CRC, London, 2013), pp. 143-180

18. R.F. Nalewajski, Struct. Bond. 149, 51 (2012)

19. R.F. Nalewajski, Sci. Technol. 1, 105 (2012)

20. R.F. Nalewajski, in Advances in Quantum Systems Research (Nova, New York, 2012), in press

21. R.F. Nalewajski, J. Math. Chem. 51, 369 (2013)

22. R.F. Nalewajski, Ann. Phys. (Leipzig) 525, 256 (2013)

23. R.F. Nalewajski, E. Switka, A. Michalak, Int. J. Quantum Chem. 87, 198 (2002)

24. R.F. Nalewajski, E. Świtka, Phys. Chem. Chem. Phys. 4, 4952 (2002)

25. R.F. Nalewajski, E. Broniatowska, J. Phys. Chem. A. 107, 6270 (2003)

26. R.F. Nalewajski, R.G. Parr, Proc. Natl. Acad. Sci. USA 97, 8879 (2000)

27. R.F. Nalewajski, R.G. Parr, J. Phys. Chem. A 105, 7391 (2001) 
28. R.F. Nalewajski, R. Loska, Theor. Chem. Acc. 105, 374 (2001)

29. R.F. Nalewajski, Chem. Phys. Lett. 372, 28 (2003)

30. R.G. Parr, P.W. Ayers, R.F. Nalewajski, J. Phys. Chem. A 109, 3957 (2005)

31. R.F. Nalewajski, Phys. Chem. Chem. Phys. 4, 1710 (2002)

32. R.F. Nalewajski, Adv. Quantum Chem. 43, 119 (2003)

33. R.F. Nalewajski, E. Broniatowska, Theor. Chem. Acc. 117, 7 (2007)

34. F.L. Hirshfeld, Theor. Chim. Acta (Berl.) 44, 129 (1977)

35. R.F. Nalewajski, Int. J. Quantum Chem. 108, 2230 (2008)

36. R.F. Nalewajski, J. Math. Chem. 47, 667 (2010)

37. R.F. Nalewajski, P. de Silva, J. Mrozek, J. Mol. Struct. THEOCHEM 954, 57 (2010)

38. R.F. Nalewajski, A.M. Köster, S. Escalante, J. Phys. Chem. A 109, 10038 (2005)

39. A.D. Becke, K.E. Edgecombe, J. Chem. Phys. 92, 5397 (1990)

40. B. Silvi, A. Savin, Nature 371, 683 (1994)

41. A. Savin, R. Nesper, S. Wengert, T.F. Fässler, Angew. Chem. Int. Ed. Engl. 36, 1808 (1997)

42. R.F. Nalewajski, J. Phys. Chem. A 104, 11940 (2000)

43. R.F. Nalewajski, J. Math. Chem. 49, 2308 (2011)

44. R.F. Nalewajski, J. Math. Chem. 51, 7 (2013)

45. R.F. Nalewajski, K. Jug, in Reviews of Modern Quantum Chemistry: A Celebration of the Contributions of Robert G. Parr, Vol. I, ed. by K.D. Sen (World Scientific, Singapore, 2002), pp. 148-203

46. R.F. Nalewajski, Struct. Chem. 15, 391 (2004)

47. R.F. Nalewajski, Mol. Phys. 102, 531, 547 (2004)

48. R.F. Nalewajski, Mol. Phys. 103, 451 (2005)

49. R.F. Nalewajski, Mol. Phys. 104, 1977, 2533 (2006)

50. R.F. Nalewajski, Theor. Chem. Acc. 114, 4 (2005)

51. R.F. Nalewajski, J. Math. Chem. 38, 43 (2005)

52. R.F. Nalewajski, J. Math. Chem. 43, 265 (2008)

53. R.F. Nalewajski, J. Math. Chem. 45, 607 (2009)

54. R.F. Nalewajski, J. Math. Chem. 45, 709 (2009)

55. R.F. Nalewajski, J. Math. Chem. 45, 776 (2009)

56. R.F. Nalewajski, J. Math. Chem. 45, 1041 (2009)

57. R.F. Nalewajski, Int. J. Quantum Chem. 109, 425, 2495 (2009)

58. R.F. Nalewajski, J. Math. Chem. 47, 709 (2010)

59. R.F. Nalewajski, J. Math. Chem. 49, 592 (2011)

60. R.F. Nalewajski, J. Math. Chem. 43, 780 (2008)

61. R.F. Nalewajski, J. Phys. Chem. A 111, 4855 (2007)

62. R.F. Nalewajski, Mol. Phys. 104, 3339 (2006)

63. R.F. Nalewajski, J. Phys. Chem. A 107, 3792 (2003)

64. R.F. Nalewajski, Mol. Phys. 104, 255 (2006)

65. R.F. Nalewajski, Ann. Phys. Leipzig 13, 201 (2004)

66. H.B. Callen, Thermodynamics: An Introduction to the Physical Theories of the Equilibrium Thermostatics and Irreversible Thermodynamics (Wiley, New York, 1960)

67. R.F. Nalewajski, Adv. Quantum Chem. 56, 217 (2009)

68. R.F. Nalewajski, D. Szczepanik, J. Mrozek, Adv. Quantum Chem. 61, 1 (2011)

69. R.F. Nalewajski, D. Szczepanik, J. Mrozek, J. Math. Chem. 50, 1437 (2012)

70. R.F. Nalewajski, J. Math. Chem. 47, 692, 808 (2010)

71. R.F. Nalewajski, J. Math. Chem. 49, 371, 546 (2011)

72. R.F. Nalewajski, P. Gurdek, J. Math. Chem. 49, 1226 (2011)

73. R.F. Nalewajski, Int. J. Quantum Chem. 112, 2355 (2012)

74. R.F. Nalewajski, P. Gurdek, Struct. Chem. 23, 1383 (2012)

75. R.F. Nalewajski, Int. J. Quantum Chem. 113, 766 (2013)

76. R.F. Nalewajski, J. Math. Chem. 49, 806 (2011)

77. S. López-Rosa, Information-Theoretic Measures of Atomic and Molecular Systems. Ph.D. Thesis, University of Granada (2010)

78. S. López-Rosa, R.O. Esquivel, J.C. Angulo, J. Antolín, J.S. Dehesa, N. Flores- Gallegos, J. Chem. Theory Comput. 6, 145 (2010)

79. R.F. Nalewajski, Quantum information descriptors and communications in molecules. J. Math. Chem. (2013), submitted 
80. R.F. Nalewajski, in Quantum Matter Chemistry, ed. by A. Herman (American Scientific Publishers, 2013), in press

81. R.F. Nalewajski, Communications in molecules: local and multi-configurational channels and their entropic descriptors of bond multiplicity and composition. J. Math. Chem. (2013), submitted

82. P.A.M. Dirac, The Principles of Quantum Mechanics, 4th edn. (Clarendon, Oxford, 1958)

83. R.F. Nalewajski, J. Math. Chem. 44, 414 (2008) 